\title{
Global Regularity of 2-D Density Patches for Viscous Inhomogeneous Incompressible Flow with General Density: High Regularity Case
}

\author{
Xian Liao ${ }^{1}$ and Ping Zhang ${ }^{2, *}$ \\ ${ }^{1}$ Institute for Analysis, Karlsruhe Institute for Technology, Englerstrasse 2, 76131 \\ Karlsruhe, Germany \\ 2 Academy of Mathematics \& Systems Science and Hua Loo-Keng Key Laboratory \\ of Mathematics, Chinese Academy of Sciences, China, and School of Mathematical \\ Sciences, University of Chinese Academy of Sciences, Beijing 100049, China
}

Received 7 September 2017; Accepted (in revised version) 8 June 2018

\begin{abstract}
This paper is a continuation work of [26] and studies the propagation of the high-order boundary regularities of the two-dimensional density patch for viscous inhomogeneous incompressible flow. We assume the initial density $\rho_{0}=\eta_{1} \mathbf{1}_{\Omega_{0}}+\eta_{2} \mathbf{1}_{\Omega_{0}^{c}}$, where $\left(\eta_{1}, \eta_{2}\right)$ is any pair of positive constants and $\Omega_{0}$ is a bounded, simply connected domain with $W^{k+2, p}\left(\mathbb{R}^{2}\right)$ boundary regularity. We prove that for any positive time $t$, the density function $\rho(t)=\eta_{1} \mathbf{1}_{\Omega(t)}+\eta_{2} \mathbf{1}_{\Omega(t)^{c}}$, and the domain $\Omega(t)$ preserves the $W^{k+2, p}$-boundary regularity.
\end{abstract}

Key Words: Inhomogeneous incompressible Navier-Stokes equations, density patch, striated distributions, Littlewood-Paley theory.

AMS Subject Classifications: 35Q30, 76D03

\section{Introduction}

We consider the two-dimensional density-dependent incompressible Navier-Stokes system:

$$
\left\{\begin{array}{l}
\partial_{t} \rho+\operatorname{div}(\rho v)=0, \\
\partial_{t}(\rho v)+\operatorname{div}(\rho v \otimes v)-\Delta v+\nabla \pi=0, \\
\operatorname{div} v=0, \\
\left.(\rho, v)\right|_{t=0}=\left(\rho_{0}, v_{0}\right) .
\end{array} \quad(t, x) \in \mathbb{R}^{+} \times \mathbb{R}^{2},\right.
$$

Here the unknowns $(\rho, v) \in \mathbb{R}^{+} \times \mathbb{R}^{2}$ represent the density and the velocity field of the two-dimensional fluid at time $t$ and point $x$ respectively, and $\pi$ designates the unknown pressure which ensures the incompressibility of the fluid.

${ }^{*}$ Corresponding author. Email addresses: xian.liao@kit.edu (X. Liao), zp@amss .ac.cn (P. Zhang) 
This system (1.1) can describe the dynamics of a viscous fluid which is incompressible but with variable density, e.g., mixtures of incompressible and non-reactant components, fluids containing a melted substance. In the simple case when $\rho_{0} \equiv 1$, the system (1.1) reduces to the classical incompressible Navier-Stokes system:

$$
\partial_{t} v+\operatorname{div}(v \otimes v)-\Delta v+\nabla \pi=0, \quad \operatorname{div} v=0,\left.\quad v\right|_{t=0}=v_{0} .
$$

There is a substantial amount of literature devoted to the study of the well-posedness issue of the system (1.1), e.g., [28,32] in the weak solution framework, $[1-3,12,24,29]$ in the strong solution framework. If the density function has a jump across some hypersurface which is of interest in this paper, $[13,14,16,22,30]$ have also established some well-posedness results (see [26] for more detailed introduction to these references).

We are interested in the propagation of regularities of the interface between fluids with different densities, for which we take the assumptions as follows. Let $\Omega_{0}$ be a simply connected bounded domain with $W^{k+2, p}\left(\mathbb{R}^{2}\right)$-boundary regularity, $k \geq 1, p \in(2,4)$, that is, we can parametrize $\partial \Omega_{0}$ as

$$
\begin{gathered}
\gamma: \mathbb{S}^{1} \mapsto \partial \Omega_{0} \text { via } s \mapsto \gamma(s) \text { with } \gamma \in\left(W^{k+2, p}\left(\mathbb{S}^{1}\right)\right)^{2} \\
\text { and } \partial_{s} \gamma(s)=X_{0}(\gamma(s)), \quad s \in \mathbb{S}^{1} .
\end{gathered}
$$

Here $X_{0}(\cdot) \in \mathbb{R}^{2}$ is a vector field defined on $\mathbb{R}^{2}$ which is tangential to $\partial \Omega_{0}$ : if $\partial \Omega_{0}=f_{0}^{-1}(0)$ is the level set then $X_{0}=\left(-\partial_{2}, \partial_{1}\right)^{T} f_{0}$. We denote by $\partial_{X_{0}} u=X_{0} \cdot \nabla u$, the directional derivative of $u$ along $X_{0}$. Then we easily calculate

$$
\partial_{s}^{2} \gamma(s)=\partial_{s}\left(X_{0}(\gamma(s))\right)=X_{0}(\gamma(s)) \cdot \nabla X_{0}(\gamma(s))=\left(\partial_{X_{0}} X_{0}\right)(\gamma(s)),
$$

and repeating this calculation gives $\partial_{s}^{\ell} \gamma(s)=\left(\partial_{X_{0}}^{\ell-1} X_{0}\right)(\gamma(s))$. Hence the boundary regularity assumption is equivalent to the following assumption on $X_{0}$ :

$$
\partial_{X_{0}}^{\ell-1} X_{0} \in\left(W^{2, p}\left(\mathbb{R}^{2}\right)\right)^{2}, \quad \ell=1, \cdots, k, \quad \operatorname{div} X_{0}=0 .
$$

For any $\eta_{1}, \eta_{2}>0$, we take the initial density $\rho_{0}$ and the initial velocity $v_{0}$ as

$$
\rho_{0}=\eta_{1} \mathbf{1}_{\Omega_{0}}+\eta_{2} \mathbf{1}_{\Omega_{0}^{c}}, \quad v_{0} \in\left(L^{2} \cap \dot{B}_{2,1}^{s_{0}}\left(\mathbb{R}^{2}\right)\right)^{2} \quad \text { and } \quad \partial_{X_{0}}^{\ell} v_{0} \in\left(L^{2} \cap \dot{B}_{2,1}^{s_{\ell}}\left(\mathbb{R}^{2}\right)\right)^{2},
$$

for some

$s_{0} \in(0,1), \quad s_{\ell}=s_{0}-\theta_{0} \ell$ with some fixed $\theta_{0} \in\left(0, s_{0} / k\right), \quad \ell=1, \cdots, k, \quad p \in\left(2,2 /\left(1-s_{k}\right)\right)$. Here we have taken $v_{0} \in\left(L^{2}\left(\mathbb{R}^{2}\right)\right)^{2}$ with finite $\left(\dot{B}_{2,1}^{s_{0}}\left(\mathbb{R}^{2}\right)\right)^{2}$-Besov norm defined as follows (see e.g., [5]):

Definition 1.1. Consider a smooth radial function $\varphi$ on $\mathbb{R}$, supported in $[3 / 4,8 / 3]$ such that $\sum_{j \in \mathbb{Z}} \varphi\left(2^{-j} \tau\right)=1$ for any $\tau>0$. We denote

$$
\Delta_{j} a=\mathcal{F}^{-1}\left(\varphi\left(2^{-j}|\xi|\right) \widehat{a}(\xi)\right), \quad j \in \mathbb{Z} .
$$


Let $(p, r, \kappa) \in[1,+\infty]^{3}$ and $s \in \mathbb{R}$. The Besov norms are defined as

$$
\|a\|_{\dot{B}_{p, r}^{s}} \stackrel{\text { def }}{=}\left\|\left(2^{j s}\left\|\Delta_{j} a\right\|_{L^{p}}\right)_{j}\right\|_{\ell^{r}(\mathbb{Z})} \quad \text { and } \quad\|a\|_{\tilde{L}_{T}^{\kappa}\left(\dot{B}_{p, r}^{s}\right)} \stackrel{\text { def }}{=}\left\|\left(2^{j s}\left\|\Delta_{j} a\right\|_{L_{t}^{\kappa}\left([0, T] ; L^{p}\right)}\right)_{j}\right\|_{\ell^{r}(\mathbb{Z})} \text {. }
$$

Similarly for $\ell=1, \cdots, k$, we have taken $\partial_{X_{0}}^{\ell} v_{0} \in\left(L^{2}\left(\mathbb{R}^{2}\right)\right)^{2}$ with finite $\left(\dot{B}_{2,1}^{s_{\ell}}\left(\mathbb{R}^{2}\right)\right)^{2}$-Besov norm. When $p=r=2$, the Besov spaces $\dot{B}_{2,2}^{s}$ coincide with the classical homogeneous Sobolev spaces $\dot{H}^{s}$. Moreover, the embedding $H^{s} \hookrightarrow L^{2} \cap \dot{B}_{2,1}^{s_{0}}$ holds whenever $0<s_{0}<s$.

Suppose that $(\rho, v)$ with $v \in L_{\text {loc }}^{1}\left(\mathbb{R}^{+} ;\left(W^{2, p}\left(\mathbb{R}^{2}\right)\right)^{2}\right)$ (and hence Lipschitzian in space when averaged in time) is the solution of the Cauchy problem (1.1)-(1.4). Let $\psi(t, \cdot) \in$ $L_{\text {loc }}^{\infty}\left(\mathbb{R}^{+} ;\left(W^{2, p}\left(\mathbb{R}^{2}\right)\right)^{2}\right)$ be the flow associated with the vector field $v: \frac{d}{d t} \psi(t, x)=v(t, \psi(t, x))$, $\psi(0, x)=x$ and let $\Omega(t)=\psi\left(t, \Omega_{0}\right)$ be the domain transported by this flow with the $W^{2, p_{-}}$ boundary parametrization $\psi\left(t, \gamma_{0}(\cdot)\right): \mathbb{S}^{1} \mapsto \partial \Omega(t)$. Then we deduce from the transport density equation of (1.1) that

$$
\rho(t, x)=\eta_{1} \mathbf{1}_{\Omega(t)}(x)+\eta_{2} \mathbf{1}_{\Omega(t) c}(x) .
$$

The tangential vector field $X(t, \cdot)$ of the boundary of the domain $\Omega(t)$ is transported by this flow in this following way

$$
X(t, \psi(t, x))=X_{0}(x) \cdot \nabla \psi(t, x),
$$

or equivalently,

$$
\left\{\begin{array}{l}
\partial_{t} X+v \cdot \nabla X=X \cdot \nabla v \\
X(0, x)=X_{0}(x)
\end{array}\right.
$$

Then the question below arises: whether or not the evolved boundary $\partial \Omega(t)$ persists the initial boundary regularity, that is, $\psi\left(t, \gamma_{0}(\cdot)\right) \in W^{k+2, p}\left(\mathrm{~S}^{1}\right), k \geq 1$ for any positive time $t$ ? Or equivalently, by view of $\partial_{s}^{\ell} \psi\left(t, \gamma_{0}(s)\right)=\left(\partial_{X}^{\ell-1} X\right)\left(t, \psi\left(t, \gamma_{0}(s)\right)\right)^{\dagger}$, we ask ourselves whether or not

$$
\left(\partial_{X}^{\ell-1} X\right) \in L_{\mathrm{loc}}^{\infty}\left(\mathbb{R}^{+} ;\left(W^{2, p}\left(\mathbb{R}^{2}\right)\right)^{2}\right) \quad \text { for } \ell=1, \cdots, k .
$$

The original question was proposed by P.-L. Lions in [28] with $\left(\eta_{1}, \eta_{2}\right)=(1,0)$. As a first progress toward this question, we [25] confirmed the propagation of the boundary regularity of any order under the small jump condition: $\left|\eta_{1}-\eta_{2}\right|$ is sufficiently small. For

\footnotetext{
${ }^{\dagger}$ We calculate directly

$$
\partial_{s}\left(\psi\left(t, \gamma_{0}(s)\right)\right)=X_{0}\left(\gamma_{0}(s)\right) \cdot \nabla \psi\left(t, \gamma_{0}(s)\right)=X\left(t, \psi\left(t, \gamma_{0}(s)\right)\right),
$$

and then recursively for any $\ell \geq 1$,

$$
\partial_{s}^{\ell}\left(\psi\left(t, \gamma_{0}(s)\right)\right)=\partial_{s}^{\ell-1}\left(\left(\partial_{X_{0}} \psi\right)\left(t, \gamma_{0}(s)\right)\right)=\cdots=\left(\partial_{X_{0}}^{\ell} \psi\right)\left(t, \gamma_{0}(s)\right)=\left(\partial_{X}^{\ell-1} X\right)\left(t, \psi\left(t, \gamma_{0}(s)\right)\right) \text {. }
$$
}


any positive numbers $\left(\eta_{1}, \eta_{2}\right)$, we [26] considered the low regularity case $k=1$ and in this paper we continue to study the high regularity case with $k \geq 2$.

Recently $[17,19]$ studied the propagation of the low boundary regularity of Hölder type. Danchin and Mucha [15] investigated the vacuum case and hence the aforementioned question has a complete answer in the low regularity regime.

We consider this regularity propagation problem in the framework of conormal distributions or striated distributions (with respect to the regularities only in the tangential direction in (1.3), (1.4), (1.8)). This idea was used by J.-Y. Chemin [8,9] to prove the global regularities of the two-dimensional vortex patch for ideal flow (see also $[6,7,10,11,18,20$, 21,31]).

In the remaining part of the introduction, we shall introduce

- The main result in Subsection 1.1,

- A series of equations in Subsection 1.2,

- Two useful lemmas in Subsection 1.3,

- The outline of the proof in Subsection 1.4,

and we will supply the detailed proofs in Section 2, following the outline in Subsection 1.4 .

\subsection{Main results}

In order to show (1.8), we have to consider $\partial_{X}^{\ell-1} X^{\prime}$ s evolutionary equation. For notational simplicity, we first introduce the following notations:

$$
\begin{aligned}
& D_{t}:=\partial_{t}+v \cdot \nabla, \quad \partial_{X}:=X \cdot \nabla, \\
& \rho^{\ell}:=\partial_{X}^{\ell} \rho, \quad v^{\ell}:=\partial_{X}^{\ell} v, \quad \pi^{\ell}:=\partial_{X}^{\ell} \pi, \quad X^{\ell}:=\partial_{X}^{\ell} X, \quad \ell=0,1, \cdots \quad \text { and } \quad \partial_{X}^{-1} u \stackrel{\text { def }}{=} 0,
\end{aligned}
$$

then Eq. (1.7) implies that the two operators $D_{t}$ and $\partial_{X}$ commute:

$$
\left[D_{t} ; \partial_{X}\right]=D_{t} X-\partial_{X} v=0 .
$$

Therefore we apply $\partial_{X}^{\ell}$ to the transport density equation $D_{t} \rho=0$ in (1.1) to obtain $D_{t} \rho^{\ell}=0$. Under the initial density patch assumption (1.4), $\partial_{X_{0}}^{\ell} \rho_{0}=0$ for any $\ell \geq 1$ and hence

$$
\rho^{\ell}(t)=\left(\partial_{X}^{\ell} \rho\right)(t)=0, \quad \forall \ell \geq 1 .
$$

Similarly we apply $\partial_{X}^{\ell-1}$ to the transport equation (1.7) $D_{t} X=\partial_{X} v$ to get the transport equation for $\partial_{X}^{\ell-1} X=X^{\ell-1}$ :

$$
\left\{\begin{array}{l}
D_{t} \mathrm{X}^{\ell-1}=\partial_{t} \mathrm{X}^{\ell-1}+v \cdot \nabla \mathrm{X}^{\ell-1}=\mathrm{v}^{\ell} \\
\mathrm{X}^{\ell-1}(0, x)=\left(\partial_{X_{0}}^{\ell-1} \mathrm{X}_{0}\right)(x) .
\end{array}\right.
$$


In order to show $\mathrm{X}^{\ell-1}(t, \cdot) \in\left(W^{2, p}\right)^{2}$, we need $\mathrm{v}^{\ell} \in L^{1}\left([0, t] ;\left(W^{2, p}\right)^{2}\right)$ and this can be obtained from the $H^{1}$-energy estimates for $\mathrm{v}^{\ell}$ and $\left(D_{t} \mathrm{v}^{\ell}\right) \ddagger \S$. To this end, we will consider the $\ell$-th unknowns $\left(\mathrm{v}^{\ell}, D_{t} \mathrm{v}^{\ell}, \nabla D_{t} \pi^{\ell}, \mathrm{X}^{\ell-1}\right)$ together and aim to get the estimate for $A_{\ell}(t)$ defined below

$$
A_{\ell}(t):=\left\|\mathrm{v}^{\ell}\right\|_{\widetilde{L}_{t}^{\infty}\left(\dot{B}_{2,1}^{s_{\ell}}\right) \cap \widetilde{L}_{t}^{2}\left(\dot{B}_{2,1}^{1+s_{\ell}}\right)}+\left\|\mathrm{X}^{\ell-1}\right\|_{L_{t}^{\infty}\left(W^{2, p}\right)}+A_{\ell 1}(t)+A_{\ell 2}(t),
$$

where

$$
\begin{aligned}
A_{\ell 1}(t):= & \left\|\mathrm{v}^{\ell}\right\|_{L_{t}^{\infty}\left(L^{2}\right) \cap L_{t}^{2}\left(\dot{H}^{1}\right)}+\left\|\sigma^{\frac{1-s_{\ell}}{2}} \nabla \mathrm{v}^{\ell}\right\|_{L_{t}^{\infty}\left(L^{2}\right)} \\
& +\left\|\sigma^{\frac{1-s_{\ell}}{2}}\left(\partial_{t} \mathrm{v}^{\ell}, \nabla^{2} \mathbf{v}^{\ell}, \nabla \pi^{\ell}\right)\right\|_{L_{t}^{2}\left(L^{2}\right)^{\prime}} \\
A_{\ell 2}(t):= & \left\|\sigma^{1-\frac{s_{\ell}}{2}}\left(D_{t} \mathrm{v}^{\ell}, \nabla^{2} \mathbf{v}^{\ell}, \nabla \pi^{\ell}\right)\right\|_{L_{t}^{\infty}\left(L^{2}\right)}+\left\|\sigma^{1-\frac{s_{\ell}}{2}} \nabla D_{t} \mathbf{v}^{\ell}\right\|_{L_{t}^{2}\left(L^{2}\right)} \\
& +\left\|\sigma^{\frac{3-s_{\ell}}{2}} \nabla D_{t} \mathbf{v}^{\ell}\right\|_{L_{t}^{\infty}\left(L^{2}\right)}+\left\|\sigma^{\frac{3-s_{\ell}}{2}}\left(D_{t}^{2} \mathbf{v}^{\ell}, \nabla^{2} D_{t} \mathbf{v}^{\ell}, \nabla D_{t} \pi^{\ell}\right)\right\|_{L_{t}^{2}\left(L^{2}\right)^{\prime}}
\end{aligned}
$$

correspond to the $H^{1}$-estimates of $\mathrm{v}^{\ell}, D_{t} \mathrm{v}^{\ell}$ respectively. In the above and in the following, the notations such as $L_{t}^{p}\left(L^{q}\right)$ will always mean $L^{p}\left([0, t] ; L^{q}\left(\mathbb{R}^{2}\right)\right)$. The $\left(L_{t}^{p}\left(L^{q}\right)\right)^{m}$-norm of the vector field $\left(u_{1}, \cdots, u_{m}\right) \in \mathbb{R}^{m}$ will be simply denoted by $\left\|\left(u_{1}, \cdots, u_{m}\right)\right\|_{L_{t}^{p}\left(L^{q}\right)}$. The notation $\sigma=\sigma(t)$ denotes the time weight

$$
\sigma=\sigma(t)=\min \{1, t\} .
$$

We [26] have studied the Cauchy problem (1.1)-(1.4) and proved (1.8) for $k=1$ by considering the coupled system (1.1)-(1.7) (see also (1.26)-(1.27) below) with the initial data (1.3)-(1.4) for $k=1$. Let us recall the global well-posedness and the low regularity propagation results therein where the estimates for $v$ also play an important role here:

Theorem 1.1. Let the initial data $\left(\rho_{0}, v_{0}, X_{0}\right)$ satisfy (1.3)-(1.4) for $k=1$. Then the coupled system (1.1)-(1.7) has a unique global-in-time solution $(\rho, v, \nabla \pi, X)$ such that the density function $\rho(t)$ is still density patch (1.5) where the domain $\Omega(t)$ preserves the same boundary regularity (1.8), and the velocity field $v(t) \in\left(L^{2} \cap \dot{B}_{2,1}^{s_{0}}\left(\mathbb{R}^{2}\right)\right)^{2}$ together with its tangential derivative $\left(\partial_{X} v\right)(t) \in$ $\left(L^{2} \cap \dot{B}_{2,1}^{s_{1}}\left(\mathbb{R}^{2}\right)\right)^{2}$ persist the initial regularities and are $\left(W^{2, p}\left(\mathbb{R}^{2}\right)\right)^{2}$ for any positive time.

Furthermore, for any $r \in[2, \infty)$ there exist some universal constant $C_{0}$ and some constant $\mathcal{C}_{1}$ (depending on the initial data $\eta_{1}, \eta_{2},\left\|v_{0}\right\|_{L^{2} \cap \dot{B}_{2,1}^{s_{0}}},\left\|X_{0}\right\|_{W^{2, p},},\left\|\partial_{X_{0}} v_{0}\right\|_{L^{2} \cap \dot{B}_{2,1}^{s_{1}}}$ ) such that

$$
A_{0}(t)+\left(\int_{0}^{t} B_{0} d t^{\prime}\right)^{\frac{1}{2}} \leq\left\|v_{0}\right\|_{L^{2} \cap \dot{B}_{2,1}^{s_{0}}} \exp \left(C_{0} \exp \left(C_{0}\left\|v_{0}\right\|_{L^{2}}^{4}\right)\right), \quad \forall t \geq 0,
$$

$\ddagger$ We consider $D_{t} \mathrm{v}^{\ell}$ instead of $\partial_{t} \mathrm{v}^{\ell}$ since we would like to benefit from the density equation $D_{t} \rho=0$ while $\partial_{t} \rho=-v \cdot \nabla \rho$ has singularities since the density $\rho$ is discontinuous in space variable. The idea was used by D. Hoff in [23] for the study of the compressible Navier-Stokes system.

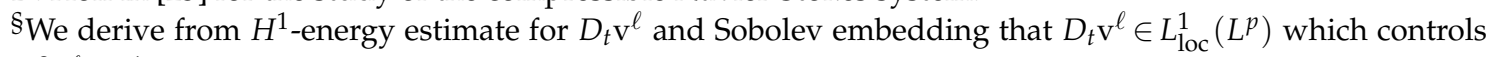
$\nabla^{2} \mathrm{v}^{\ell} \in L_{\mathrm{loc}}^{1}\left(L^{p}\right)$ up to lower order terms, see Eq. (1.29) below. 
where (recalling the time weight $\sigma(t)$ in (1.15))

$$
\begin{aligned}
A_{0}(t):= & \|v\|_{L_{t}^{\infty}\left(L^{2}\right)}+\|v\|_{\tilde{L}_{t}^{\infty}\left(\dot{B}_{2,1}^{s_{0}}\right) \cap \widetilde{L}_{t}^{2}\left(\dot{B}_{2,1}^{1+s_{0}}\right)}+\left\|\sigma^{\frac{1-s_{0}}{2}} v\right\|_{\tilde{L}_{t}^{\infty}\left(\dot{B}_{2,1}^{1}\right)}+\left\|\sigma^{1-\frac{s_{0}}{2}} \nabla v\right\|_{L_{t}^{\infty}\left(L^{\infty} \cap L^{\frac{2 p}{p-2}}\right)} \\
& +\left\|\sigma^{\left(\frac{3}{2}-\frac{1}{r}-\frac{s_{0}}{2}\right)}\left(D_{t} v, \partial_{t} v, v \otimes \nabla v, \nabla^{2} v, \nabla \pi\right)\right\|_{L_{t}^{\infty}\left(L^{r}\right)} \\
& +\left\|\sigma^{\frac{3-s_{0}}{2}}\left(\nabla D_{t} v, D_{t} \nabla v, \nabla \partial_{t} v\right)\right\|_{L_{t}^{\infty}\left(L^{2}\right)} \\
& +\left\|\sigma^{1-\frac{1}{p}-\frac{s_{0}}{2}} \nabla v\right\|_{L_{t}^{\infty}\left(L^{p}\right)}+\left\|\sigma^{1-\frac{s_{0}}{2}}\left(D_{t} v, \partial_{t} v, v \otimes \nabla v, \nabla^{2} v, \nabla \pi\right)\right\|_{L_{t}^{4}\left(L^{4}\right)}, \\
B_{0}(t):= & \sum_{m=2,4, p, \frac{2 p}{p-2}}\left(\|\nabla v(t)\|_{L^{2}}^{2}+\left\|\sigma^{\left(1-\frac{1}{m}-\frac{s_{0}}{2}\right)}\left(\partial_{t} v, \nabla^{2} v, \nabla \pi, D_{t} v, v \otimes \nabla v\right)(t)\right\|_{L^{m}}^{2}\right. \\
& +\left\|\sigma^{1-\frac{s_{0}}{2}} \nabla \partial_{t} v(t)\right\|_{L^{2}}^{2}+\left\|\sigma^{\frac{1-s_{0}}{2}} \nabla v(t)\right\|^{2} \\
& +\left\|\sigma^{\frac{3-s_{0}}{2}}\left(D_{t}^{2} v, \nabla^{2} D_{t} v, D_{t} \nabla^{2} v, \nabla D_{t} \pi, D_{t} \nabla \pi, v \cdot\left(\nabla v_{t}+D_{t} \nabla v\right), D_{t} v \cdot \nabla v\right)(t)\right\|_{L^{2}}^{2} \\
& \left.+\left\|\sigma^{\left(\frac{3}{2}-\frac{1}{m}-\frac{s_{0}}{2}\right.}\left(D_{t} v, v \otimes \nabla v, \partial_{t} v\right)(t)\right\|_{L^{\infty}}^{2}\left(\nabla D_{t} v, D_{t} \nabla v, v \otimes \nabla^{2} v, \nabla v \otimes \nabla v, \nabla \partial_{t} v\right)(t) \|_{L^{m}}^{2}\right)
\end{aligned}
$$

and (recalling the definition (1.13) of $A_{\ell}$ )

$$
A_{1}(t) \leq \exp \left(\exp \left(\mathcal{C}_{1}\langle t\rangle^{2}\right)\right), \quad\langle t\rangle:=\max \{1, t\} .
$$

Here the notation $a \otimes b$ means: $(a \otimes b)_{i j}=a_{i} b_{j}$ for $a=\left(a_{i}\right)_{i}$ and $b=\left(b_{j}\right)_{j}$.

In this paper we will generalize $k=1$ in Theorem 1.1 to any positive integer and the main result of this paper is the following:

Theorem 1.2. Let $k \geq 1$ be any integer. Let the initial data $\left(\rho_{0}, v_{0}, X_{0}\right)$ be given by (1.3)-(1.4). Then the coupled system (1.1)-(1.7) has a unique solution $(\rho, v, \nabla \pi, X)$ such that the density patch (1.5) persists the initial boundary regularity (1.8).

Furthermore, there exists a constant $\mathcal{C}_{\ell}$ (depending on the initial data (1.3)-(1.4)) such that (recalling the definition (1.13) of $A_{\ell}$ and $\langle t\rangle=\max \{1, t\}$ )

$$
A_{\ell}(t) \leq \mathcal{H}_{\ell}(t):=\exp \exp \exp \cdots \exp \left(\mathcal{C}_{\ell}\langle t\rangle^{2}\right) \text { with }(\ell+1) \text {-times } \exp , \quad \ell=1, \cdots, k \text {. }
$$

Remark 1.1. The initial density assumption in (1.4) can be relaxed to more general case:

$$
0<\rho_{*} \leq \rho_{0} \leq \rho^{*}, \quad \partial_{X_{0}}^{\ell} \rho_{0} \in L^{\infty}\left(\mathbb{R}^{2}\right), \quad \ell=1, \cdots, k .
$$

\subsection{A series of equations}

Let $(\rho, v, \nabla \pi, X)$ be the solution of the Cauchy problem (1.1)-(1.7) and (1.3)-(1.4) given by Theorem 1.1. Recall the notations $D_{t}, \mathrm{v}^{\ell}, \nabla \pi^{\ell}, \mathrm{X}^{\ell}$ defined in (1.9). In this subsection we 
will derive the system satisfied by $\left(\mathrm{v}^{\ell}, \nabla \pi^{\ell}\right)$, which will be of the following form:

$$
\left\{\begin{array}{l}
\rho \partial_{t} u+\rho v \cdot \nabla u-\Delta u+\nabla \Pi=f, \quad(t, x) \in \mathbb{R}^{+} \times \mathbb{R}^{2}, \\
\operatorname{div} u=\operatorname{div} g, \\
\left.u\right|_{t=0}=u_{0},
\end{array}\right.
$$

where $(u, \nabla \Pi)$ are the unknowns and $\left(f, g, u_{0}\right)$ are known data. While $\left(D_{t} \mathrm{v}^{\ell}, \nabla D_{t} \pi^{\ell}\right)$ satisfies a linear system reading as below

$$
\left\{\begin{array}{l}
\rho D_{t}\left(D_{t} \mathrm{w}\right)-\Delta\left(D_{t} \mathrm{w}\right)+\nabla \mathrm{q}=\mathrm{F}, \\
\operatorname{div} D_{t} \mathrm{w}=\operatorname{div} \mathfrak{a} \text { and } \operatorname{div} D_{t}\left(D_{t} \mathrm{w}\right)=\operatorname{div} \mathfrak{b},
\end{array} \quad(t, x) \in \mathbb{R}^{+} \times \mathbb{R}^{2},\right.
$$

where $\left(D_{t} \mathrm{w}, \nabla \mathrm{q}\right)$ are the unknowns and $(\mathrm{F}, \mathfrak{a}, \mathfrak{b})$ are known data. Indeed, we will derive below the precise formulations for

$$
(f, g)=\left(f_{\ell}, g_{\ell}\right) \text { when }(u, \nabla \Pi)=\left(\mathrm{v}^{\ell}, \pi^{\ell}\right) \text {, see (1.28)-(1.29) below, }
$$

and $(\mathrm{F}, \mathfrak{a}, \mathfrak{b})=\left(\mathrm{F}_{\ell}, \mathfrak{a}_{\ell}, \mathfrak{b}_{\ell}\right)$ when $\left(D_{t} \mathrm{w}, \nabla \mathrm{q}\right)=\left(D_{t} \mathrm{v}^{\ell}, \nabla D_{t} \pi^{\ell}\right)$, see (1.31)-(1.32) below.

And we will make use of Lemmas 1.1, 1.2 below to get the energy estimates for $\mathrm{v}^{\ell}, D_{t} \mathrm{v}^{\ell}$ respectively and finally derive (1.8) for $\mathrm{X}^{\ell-1}$ which satisfies the transport equation (1.12).

We first deduce from the transport equation (1.7) and $\operatorname{div} v=0$ that $X^{\prime}$ s divergence $\operatorname{div} X$ satisfies also the free transport equation $\partial_{t}(\operatorname{div} X)+v \cdot \nabla(\operatorname{div} X)=0$. We then conclude from the initial assumption (1.3) on $X_{0}$ that the divergence-free condition always holds true for the vector field $X(t)$ :

$$
\operatorname{div} X(t, \cdot)=0, \quad \forall t \geq 0 .
$$

It is hence convenient to define the operator

$$
P_{X}=\left(\partial_{X}-(\nabla X)^{T} \cdot\right),
$$

such that due to $\operatorname{div} X=0$,

$$
\operatorname{div} P_{X} u=\operatorname{div}\left(\partial_{X} u-u \cdot \nabla X\right)=\partial_{X} \operatorname{div} u=\operatorname{div}(X \operatorname{div} u), \quad \forall u \in \mathbb{R}^{2} .
$$

1.2.1 Equations for $(v, \nabla \pi) \&\left(D_{t} v, \nabla D_{t} \pi\right)$

It is straightforward to see from (1.1) that $(v, \nabla \pi)$ satisfies $(1.21)$ with $\left(f, g, u_{0}\right)=\left(0,0, v_{0}\right)$.

We apply $D_{t}$ to the momentum equation in (1.1) to get the Eq. (1.22) for $\left(D_{t} v, \nabla D_{t} \pi\right)$ with the data $(\mathrm{F}, \mathfrak{a}, \mathfrak{b})=\left(\mathrm{F}_{0}(v, \pi), \mathfrak{a}_{0}, \mathfrak{b}_{0}\right)$ ฯ (see also [26])

$$
\left\{\begin{array}{c}
\rho D_{t}^{2} v-\Delta D_{t} v+\nabla D_{t} \pi=\mathrm{F}_{0}(v, \pi) \quad \text { with } \\
\mathrm{F}_{0}(u, \Pi)=-2 \nabla v_{\alpha} \cdot \partial_{\alpha} \nabla u-\Delta v \cdot \nabla u+\nabla v_{\alpha} \partial_{\alpha} \Pi, \\
\operatorname{div} D_{t} v=\operatorname{div} \mathfrak{a}_{0}, \quad \mathfrak{a}_{0}=v \cdot \nabla v \\
\operatorname{div} D_{t}^{2} v=\operatorname{div} \mathfrak{b}_{0}, \quad \mathfrak{b}_{0}=v \cdot\left(\nabla v_{t}+D_{t} \nabla v\right)+D_{t} v \cdot \nabla v .
\end{array}\right.
$$

Here and in what follows, repeated indices of $\alpha$ means summation of $\alpha$ from 1 to 2 .

IT The formulae for $\mathfrak{a}_{\ell}, \mathfrak{b}_{\ell}$ come from the facts that $\operatorname{div} X=\operatorname{div} v=0$ and $\left[D_{t} ; \partial_{X}\right]=0$. 
1.2.2 Equations for $\left(\mathrm{v}^{1}, \nabla \pi^{1}\right) \&\left(D_{t} \mathrm{v}^{1}, \nabla D_{t} \pi^{1}\right)$

We apply $\partial_{X}$ to the $v$-equation in (1.1) to get the Eq. (1.21) for $\mathrm{v}^{1}$ with data $\left(f_{1}, g_{1}\right)$ (see also [26])

$$
\left\{\begin{array}{c}
\rho \partial_{t} \mathrm{v}^{1}+\rho v \cdot \nabla \mathrm{v}^{1}-\Delta \mathrm{v}^{1}+\nabla \pi^{1}=f_{1}(v, \pi) \quad \text { with } \\
f_{1}(v, \pi)=-\left(\Delta X \cdot \nabla v+2 \partial_{\alpha} X \cdot \nabla \partial_{\alpha} v\right)+\nabla X^{\alpha} \partial_{\alpha} \pi, \\
\operatorname{div}^{1}=\operatorname{div} g_{1} \quad \text { with } \quad g_{1}=v \cdot \nabla X,\left.\quad \mathrm{v}^{1}\right|_{t=0}=\partial_{X_{0}} v_{0} .
\end{array}\right.
$$

We apply the operator $D_{t}$ to the $\mathrm{v}^{1}$ equation of (1.26) to get (see also [26])

$$
\left\{\begin{array}{l}
\rho D_{t}^{2} \mathrm{v}^{1}-\Delta D_{t} \mathrm{v}^{1}+\nabla D_{t} \pi^{1}=\mathrm{F}_{0}\left(\mathrm{v}^{1}, \pi^{1}\right)+D_{t} f_{1}(v, \pi):=\mathrm{F}_{1}, \\
\operatorname{div} D_{t} \mathrm{v}^{1}=\mathfrak{a}_{1} \quad \text { with } \mathfrak{a}_{1}:=D_{t} v \cdot \nabla X+P_{X}(v \cdot \nabla v), \\
\operatorname{div} D_{t}^{2} \mathbf{v}^{1}=\mathfrak{b}_{1} \quad \text { with } \quad \mathfrak{b}_{1}:=D_{t}^{2} v \cdot \nabla X+P_{X} \mathfrak{b}_{0},
\end{array}\right.
$$

where $\mathrm{F}_{0}(\cdot, \cdot), f_{1}(\cdot, \cdot)$ and $P_{X}$ are given in (1.25), (1.26) and (1.24) respectively.

\subsubsection{Equations for $\left(\mathrm{v}^{\ell}, \nabla \pi^{\ell}\right) \&\left(D_{t} \mathrm{v}^{\ell}, \nabla D_{t} \pi^{\ell}\right)$ with general $\ell \geq 2$}

Recall the definition of the operator $P_{X}$ in (1.24). Recall the definition of $g_{1}$ in (1.26). We derive $g_{\ell}$ by induction:

$$
\begin{gathered}
\operatorname{div} g_{\ell}=\operatorname{div} \mathrm{v}^{\ell}=\operatorname{div}\left(\partial_{X} \mathrm{v}^{\ell-1}\right)=\operatorname{div}\left(\mathrm{v}^{\ell-1} \cdot \nabla X+X \operatorname{div} g_{\ell-1}\right), \\
\text { with } \quad g_{\ell}:=\mathrm{v}^{\ell-1} \cdot \nabla X+P_{X} g_{\ell-1}=\sum_{i=0}^{\ell-1} P_{X}^{i}\left(\mathrm{v}^{\ell-1-i} \cdot \nabla X\right) .
\end{gathered}
$$

We apply $\partial_{X}^{\ell-1}$ to (1.26) to get inductively the Eq. (1.21) for $\left(\mathrm{v}^{\ell}, \nabla \pi^{\ell}\right)$ with data $\left(f_{\ell}, g_{\ell}\right)$ :

$$
\rho \partial_{t} \mathrm{v}^{\ell}+\rho v \cdot \nabla \mathrm{v}^{\ell}-\Delta \mathrm{v}^{\ell}+\nabla \pi^{\ell}=f_{1}\left(\mathrm{v}^{\ell-1}, \pi^{\ell-1}\right)+\partial_{X} f_{\ell-1}(v, \pi):=f_{\ell}(v, \pi),
$$

where by induction $f_{\ell}(v, \pi)=\sum_{i=0}^{\ell-1} \partial_{X}^{i} f_{1}\left(\mathrm{v}^{\ell-1-i}, \pi^{\ell-1-i}\right)$ and more precisely

$$
\begin{aligned}
f_{\ell}(v, \pi)= & \sum_{i=0}^{\ell-1} \sum_{j=0}^{i} C_{i}^{j}\left(-2 \partial_{X}^{j} \partial_{\alpha} X \cdot \partial_{X}^{i-j} \nabla \partial_{\alpha} \mathrm{v}^{\ell-1-i}\right. \\
& \left.-\partial_{X}^{j} \Delta X \cdot \partial_{X}^{i-j} \nabla \mathrm{v}^{\ell-1-i}+\partial_{X}^{j} \nabla X \cdot \partial_{X}^{i-j} \nabla \pi^{\ell-1-i}\right) .
\end{aligned}
$$

Inductively, we deduce from $\operatorname{div} \mathfrak{a}_{\ell}=\operatorname{div} D_{t} \mathrm{v}^{\ell}=\operatorname{div}\left(\partial_{X} D_{t} \mathrm{v}^{\ell-1}\right), \operatorname{div} \mathfrak{b}_{\ell}=\operatorname{div} D_{t}^{2} \mathrm{v}^{\ell}=$ $\operatorname{div}\left(\partial_{X} D_{t}^{2} \mathrm{v}^{\ell-1}\right)$ and the definitions of $P_{X},\left(\mathfrak{a}_{0}, \mathfrak{b}_{0}\right)$ in (1.24), (1.25) respectively that

$$
\begin{aligned}
& \mathfrak{a}_{\ell}:=D_{t} \mathbf{v}^{\ell-1} \cdot \nabla X+P_{X} \mathfrak{a}_{\ell-1}=\sum_{i=0}^{\ell-1} P_{X}^{i}\left(D_{t} \mathbf{v}^{\ell-1-i} \cdot \nabla X\right)+P_{X}^{\ell}(v \cdot \nabla v), \\
& \mathfrak{b}_{\ell}=D_{t}^{2} \mathbf{v}^{\ell-1} \cdot \nabla X+P_{X} \mathfrak{b}_{\ell-1}=\sum_{i=0}^{\ell-1} P_{X}^{i}\left(D_{t}^{2} \mathbf{v}^{\ell-1-i} \cdot \nabla X\right)+P_{X}^{\ell} \mathfrak{b}_{0} .
\end{aligned}
$$


We apply the operator $D_{t}$ to (1.29) to get the Eq. (1.22) for $\left(D_{t} \mathrm{v}^{\ell}, \nabla D_{t} \pi^{\ell}\right)$ with data $\left(\mathrm{F}_{\ell}, \mathfrak{a}_{\ell}, \mathfrak{b}_{\ell}\right)$ :

$$
\rho D_{t}^{2} \mathrm{v}^{\ell}-\Delta D_{t} \mathrm{v}^{\ell}+\nabla D_{t} \pi^{\ell}=\mathrm{F}_{0}\left(\mathrm{v}^{\ell}, \pi^{\ell}\right)+D_{t} f_{\ell}(v, \pi):=\mathrm{F}_{\ell},
$$

where $\mathrm{F}_{0}(\cdot, \cdot)$ is given by $(1.25)$ and hence

$$
\begin{aligned}
\mathrm{F}_{\ell}=- & 2 \partial_{\alpha} v \cdot \nabla \partial_{\alpha} \mathrm{v}^{\ell}-\Delta v \cdot \nabla \mathrm{v}^{\ell}+\nabla v \cdot \nabla \pi^{\ell}+\sum_{i=0}^{\ell-1} \sum_{j=0}^{i} C_{i}^{j} \\
& \times\left(-D_{t}\left(2 \partial_{X}^{j} \partial_{\alpha} X \cdot \partial_{X}^{i-j} \nabla \partial_{\alpha} \mathrm{v}^{\ell-1-i}+\partial_{X}^{j} \Delta X \cdot \partial_{X}^{i-j} \nabla \mathrm{v}^{\ell-1-i}\right.\right. \\
& \left.\left.-\partial_{X}^{j} \nabla X \cdot \partial_{X}^{i-j} \nabla \pi^{\ell-1-i}\right)\right) .
\end{aligned}
$$

\subsection{Two useful lemmas}

In this subsection we recall two useful lemmas in [26], which give the $H^{1}$ estimates for $u$, $D_{t} \mathrm{w}$ of the linear equations (1.21), (1.22) respectively.

Lemma 1.1. Let $(\rho, v)$ be the solution of (1.1)-(1.4). Let $(u, \nabla \Pi)$ be a smooth enough solution of (1.21) with initial data $u_{0}=0$. Then for any $s \in(0,1)$ and $\delta \in(s, 1)$, one has

$$
\begin{aligned}
& \left\|\sigma^{\frac{1-s}{2}} \nabla u\right\|_{L_{t}^{\infty}\left(L^{2}\right)}^{2}+\left\|\sigma^{\frac{1-s}{2}}\left(\partial_{t} u, \nabla^{2} u, \nabla \Pi\right)\right\|_{L_{t}^{2}\left(L^{2}\right)}^{2} \\
& \leq \mathcal{C}_{0}\left(\frac{\langle t\rangle}{\delta-s}\|g(0)\|_{\dot{B}_{2,1}^{\delta}}^{2}+\left\|\left(\sigma^{-\frac{1-\left(s_{0}-s\right)}{2}} \nabla g, \sigma^{-\frac{s}{2}}\left(f, \partial_{t} g\right)\right)\right\|_{L_{t}^{1}\left(L^{2}\right)}^{2}\right. \\
& \left.\quad+\left\|\left(\sigma^{-\frac{s}{2}} \nabla g, \sigma^{\frac{1-s}{2}}\left(\partial_{t} g, \nabla \operatorname{div} g, f\right)\right)\right\|_{L_{t}^{2}\left(L^{2}\right)}^{2}\right) .
\end{aligned}
$$

Here $\sigma=\min \{1, t\},\langle t\rangle=\max \{1, t\}$ and $\mathcal{C}_{0}=\left(1+\left\|v_{0}\right\|_{L^{2} \cap \dot{B}_{2,1}^{s_{0}}}\right)^{2} \exp \left(C_{0} \exp \left(C_{0}\left\|v_{0}\right\|_{L^{2}}^{4}\right)\right)$.

Lemma 1.2. Let $(\rho, v)$ be the solution of (1.1)-(1.4). Let $(\mathrm{w}, \nabla \mathrm{q})$ be a smooth enough solution of the system (1.22). Then for any $s \in(0,1)$, we have

$$
\begin{aligned}
& \left\|\sigma^{1-\frac{s}{2}} D_{t} \mathbf{w}\right\|_{L_{t}^{\infty}\left(L^{2}\right) \cap L_{t}^{2}\left(\dot{H}^{1}\right)}^{2}+\left\|\sigma^{\frac{3-s}{2}} \nabla D_{t} \mathbf{w}\right\|_{L_{t}^{\infty}\left(L^{2}\right)}^{2}+\left\|\sigma^{\frac{3-s}{2}}\left(D_{t}^{2} \mathbf{w}, \nabla^{2} D_{t} \mathbf{w}, \nabla \mathfrak{q}\right)\right\|_{L_{t}^{2}\left(L^{2}\right)}^{2} \\
\leq & C \exp \left(C\left\|v_{0}\right\|_{L^{2}}^{2}\right)\left\|\left(\sigma^{\frac{1-s}{2}}\left(D_{t} \mathbf{w}, \mathfrak{a}\right), \sigma^{1-\frac{s}{2}} \nabla \mathfrak{a}, \sigma^{\frac{3-s}{2}}(\nabla \operatorname{div} \mathfrak{a}, \mathfrak{b}, \mathrm{F})\right)\right\|_{L_{t}^{2}\left(L^{2}\right)^{\prime}}^{2}
\end{aligned}
$$

and in particular, for any $\varepsilon>0$, there exists $C_{\varepsilon}$ such that

$$
\begin{gathered}
\left\|\sigma^{1-\frac{s}{2}} \nabla D_{t} \mathrm{w}\right\|_{L_{t}^{2}\left(L^{2}\right)}^{2} \leq \varepsilon\left\|\sigma^{\frac{3-s}{2}} D_{t}^{2} \mathrm{w}\right\|_{L_{t}^{2}\left(L^{2}\right)}^{2}+C_{\varepsilon}\left\|\sigma^{\frac{1-s}{2}}\left(D_{t} \mathrm{w}, \mathfrak{a}\right)\right\|_{L_{t}^{2}\left(L^{2}\right)}^{2} \\
+C_{\varepsilon}\left\|\sigma^{\frac{3-s}{2}}(\mathrm{~F}, \nabla \operatorname{div} \mathfrak{a})\right\|_{L_{t}^{2}\left(L^{2}\right)}^{2}
\end{gathered}
$$




\subsection{Outline of the proof}

We prove Theorem 1.2 by an inductive argument: By Theorem 1.1, we can assume inductively that

$$
A_{l}(t) \leq \mathcal{H}_{l}(t) \text { for any } l \leq \ell-1 \text { and } \ell \leq k,
$$

and it suffices to show (1.19) $A_{\ell}(t) \leq \mathcal{H}_{\ell}(t)$. Recall the definition (1.13) of $A_{\ell}$ and

$$
\mathcal{H}_{l}(t)=\exp \operatorname{expexp} \cdots \exp \left(\mathcal{C}_{l}\langle t\rangle^{2}\right) \quad \text { with } \quad(l+1) \text {-times exp, }
$$

and the constant $\mathcal{C}_{l}$ depending on the initial data (1.3)-(1.4) until $l$-th order.

We apply Lemmas 1.1, 1.2 to the Eqs. (1.29), (1.32) respectively to get $H^{1}$ estimates for $\mathrm{v}^{\ell}, D_{t} \mathrm{v}^{\ell}$ where we take use of the inductive assumption (1.37) to control the $\ell$-th data $\left(f_{\ell}, g_{\ell}\right),\left(\mathrm{F}_{\ell}, \mathfrak{a}_{\ell}, \mathfrak{b}_{\ell}\right)$. More precisely we follow the following procedure to prove (1.19) and detailed proofs are provided in Section 2:

- We derive more estimates from the inductive assumption (1.37) in Subsection 1.4.1,

- We derive the estimates for the data $\left(f_{\ell}, g_{\ell}\right)$ in Subsection 1.4.2,

- We derive the $H^{1}$ estimate for $\mathrm{v}^{\ell}$ and then some consequent estimates for $D_{t} \mathrm{v}^{\ell}$ in Subsection 1.4.3,

- We derive the estimates for the data $\left(\mathrm{F}_{\ell}, \mathfrak{a}_{\ell}, \mathfrak{b}_{\ell}\right)$ in Subsection 1.4.4,

- We derive $H^{1}$ energy estimate of $D_{t} \mathrm{v}^{\ell}$ and finally (1.19) in Subsection 1.4.5.

\subsubsection{Deductive estimates from inductive assumptions}

Observe from Subsection 1.2.3 that for $\ell \geq 1,0 \leq i \leq \ell-1, i_{1}+\cdots+i_{r}=i$,

- $f_{\ell}$ contains terms such as $\nabla^{2} \mathrm{X}^{i} \otimes \nabla \mathrm{v}^{\ell-1-i}, \nabla \mathrm{X}^{i} \cdot \nabla^{2} \mathrm{v}^{\ell-1-i}, \nabla \mathrm{X}^{i} \cdot \nabla \pi^{\ell-1-i}$, and $g_{\ell}$ contains terms such as $\mathrm{v}^{\ell-1-i} \cdot \nabla \mathrm{X}^{i_{1}} \ldots \nabla \mathrm{X}^{i_{r}}$;

- $\mathrm{F}_{\ell}$ contains terms such as

$$
\nabla v \cdot \nabla^{2} \mathbf{v}^{\ell}, \nabla v \cdot \nabla \pi^{\ell}, \nabla^{2} v \cdot \nabla \mathbf{v}^{\ell}, D_{t}\left(\nabla^{2} X^{i} \cdot \nabla \mathbf{v}^{\ell-1-i}\right), D_{t}\left(\nabla X^{i} \cdot \nabla^{2} \mathbf{v}^{\ell-1-i}\right),
$$

$\mathfrak{a}_{\ell}$ contains terms such as $\left(D_{t} \mathrm{v}^{\ell-1-i}, \partial_{X}^{\ell-i}(v \cdot \nabla v)\right) \cdot \nabla X^{i_{1}} \ldots \nabla X^{i_{r}}$, and $\mathfrak{b}_{\ell}$ contains terms such as $\left(D_{t}^{2} \mathrm{v}^{\ell-1-i}, \partial_{X}^{\ell-i}\left(v \cdot D_{t} \nabla v, D_{t} v \cdot \nabla v, v \cdot \nabla(v \cdot \nabla v)\right)\right) \cdot \nabla X^{i_{1}} \cdots \nabla X^{i_{r}}$.

Since the operators $\partial_{X}, D_{t}$ do not commute with $\nabla, \partial_{t}$ :

$$
\left[\partial_{X} ; \nabla\right]=-\nabla X^{\alpha} \partial_{\alpha}, \quad\left[\partial_{X} ; \partial_{t}\right]=-\partial_{t} X^{\alpha} \partial_{\alpha}, \quad\left[D_{t} ; \nabla\right]=-\nabla v^{\alpha} \partial_{\alpha}, \quad\left[D_{t} ; \partial_{t}\right]=-\partial_{t} v^{\alpha} \partial_{\alpha},
$$

in order to control the $\ell$-th data $\left(f_{\ell}, g_{\ell}\right),\left(\mathrm{F}_{\ell}, \mathfrak{a}_{\ell}, \mathfrak{b}_{\ell}\right)$, we have to derive more general inductive assumptions from (1.37) which contain terms of more general form such as $\partial_{X}^{j} \nabla \partial_{X}^{k} \nabla X^{l}$, $\partial_{X}^{j} \nabla \partial_{X}^{k} \nabla \mathrm{v}^{l}, \partial_{X}^{j} \nabla D_{t} \mathbf{v}^{l}, \cdots$ 
Lemma 1.3 (Inductive assumptions). Let $(\rho, v, \nabla \pi, X)$ be the solution of the coupled system (1.1)-(1.7) with the initial data (1.3)-(1.4), such that the inductive assumption (1.37) holds. Then one has

$$
R_{l}(t)+\mathfrak{A}_{l}(t)+\int_{0}^{t} \mathfrak{B}_{l}\left(t^{\prime}\right) d t^{\prime} \leq \mathcal{H}_{l}(t) \quad \text { for } 1 \leq l \leq \ell-1
$$

In the above, for $\ell \geq 1$,

$$
R_{\ell}(t):=\sum_{m+n+\kappa \leq \ell-1}\left(\left\|\partial_{X}^{m} \nabla X^{n}\right\|_{L_{t}^{\infty}\left(W^{1, p}\right)}+\left\|\partial_{X}^{m} \nabla \partial_{X}^{n} \nabla X^{\kappa}\right\|_{L_{t}^{\infty}\left(L^{p}\right)}\right) .
$$

For

$$
\begin{aligned}
& r_{1} \in\{2,2 p /(p-2),+\infty\}, \quad r \in\{2, p\}, \\
& r_{2} \in\{2 p /(p-2),+\infty\}, \text { and } r_{3} \in\{2,2 p /(p-2)\}
\end{aligned}
$$

and $0<\varepsilon_{0}<\min \left(s_{k} / 2,(p / 2-1)\left(1-s_{0}\right)\right)$, we denote

$$
\begin{aligned}
\dot{\mathfrak{A}}_{\ell}(t):= & \sum_{\substack{i+j+l=\ell \\
m+n=\ell-1}}\left(\left\|\mathrm{v}^{i}\right\|_{L_{t}^{\infty}\left(L^{2}\right)}+\left\|\sigma^{\frac{1-s_{\ell}}{2}} \mathrm{v}^{i}\right\|_{L_{t}^{\infty}\left(L^{\frac{2 p}{p-2}}\right)}+\left\|\sigma^{\frac{1+\varepsilon_{0}-s_{\ell}}{2}}\left(\mathrm{v}^{i}, \partial_{X}^{m} \partial_{t} X\right)\right\|_{L_{t}^{\infty}\left(L^{\infty}\right)}\right. \\
& +\left\|\sigma^{\frac{1-s_{\ell}}{2}} \partial_{X}^{m} \nabla \partial_{t} X\right\|_{L_{t}^{\infty}\left(L^{2}\right)}+\left\|\sigma^{1-\frac{s_{\ell}}{2}}\left(\partial_{X}^{i} \partial_{t} \mathrm{v}^{j}, \partial_{X}^{m} D_{t} \nabla^{2} X^{n}\right)\right\|_{L_{t}^{\infty}\left(L^{2}\right)} \\
& +\left\|\sigma^{\left(1-\frac{1}{r_{1}}-\frac{s_{\ell}}{2}\right)} \partial_{X}^{i} \nabla \mathbf{v}^{j}\right\|_{L_{t}^{\infty}\left(L^{r_{1}}\right)}+\left\|\sigma^{\left(1-\frac{1}{r_{2}}-\frac{s_{\ell}}{2}\right)} \partial_{X}^{m} D_{t} \nabla X^{n}\right\|_{L_{t}^{\infty}\left(L^{r_{2}}\right)} \\
& \left.+\left\|\sigma^{\left(\frac{3}{2}-\frac{1}{r}-\frac{s_{\ell}}{2}\right)}\left(\partial_{X}^{i} \nabla \partial_{X}^{j} \nabla \mathbf{v}^{l}, D_{t} \mathbf{v}^{i}, \partial_{X}^{i} \nabla \pi^{j}\right)\right\|_{L_{t}^{\infty}\left(L^{r}\right)}\right)
\end{aligned}
$$

and

$$
\begin{aligned}
\dot{\mathfrak{B}}_{\ell}(t):= & \sum_{i+j=\ell}\left(\left\|\sigma^{\left(\frac{3}{2}-\frac{1}{r_{3}}-\frac{s_{\ell}}{2}\right)}\left(\partial_{X}^{i} \nabla D_{t} \mathbf{v}^{j}, \partial_{X}^{i} D_{t} \nabla \mathbf{v}^{j}\right)(t)\right\|_{L^{r_{3}}}^{2}+\left\|\sigma^{\left(\frac{1}{2}+\frac{1}{p}-\frac{s_{\ell}}{2}\right)} D_{t} \mathrm{v}^{i}(t)\right\|_{L^{\frac{2 p}{p-2}}}^{2}\right. \\
& \left.+\left\|\sigma^{\frac{3-s_{\ell}}{2}}\left(D_{t}^{2} \mathbf{v}^{i}, \partial_{X}^{i} D_{t} \nabla^{2} \mathbf{v}^{j}, \nabla \partial_{X}^{i} \nabla D_{t} \mathbf{v}^{j}, \partial_{X}^{i} D_{t} \nabla \pi^{j}\right)(t)\right\|_{L^{2}}^{2}\right)
\end{aligned}
$$

and

$$
\mathfrak{A}_{\ell}(t) \stackrel{\text { def }}{=} \sum_{l \leq \ell} \dot{\mathfrak{A}}_{l}(t) \quad \text { and } \quad \mathfrak{B}_{\ell}(t) \stackrel{\text { def }}{=} B_{0}(t)+\sum_{l \leq \ell} \dot{\mathfrak{B}}_{l}(t)
$$

where $B_{0}(t)$ is given by $(1.17 \mathrm{~b})$. 


\subsubsection{Estimates for $\left(f_{\ell}, g_{\ell}\right)$}

We derive in this step the estimates for the data $\left(f_{\ell}, g_{\ell}\right)$ from those estimates (1.38).

Lemma 1.4 (Estimates for $f_{\ell}$ ). Let $\ell=2, \cdots, k$, and $r \in\{2, p\}$. Under the same hypotheses in Lemma 1.3, there hold

$$
\begin{aligned}
& \left\|\sigma^{\left(\frac{3}{2}-\frac{1}{r}-\frac{s_{\ell-1}}{2}\right)}\left(f_{\ell}, \nabla \operatorname{divv}^{\ell}\right)(t)\right\|_{L^{r}} \leq \mathcal{H}_{\ell-1}(t)\left(1+\left\|\nabla X^{\ell-1}\right\|_{W^{1, p}}\right), \\
& \left\|\sigma^{\left(\frac{3}{2}-\frac{1}{r}-\frac{s_{\ell}}{2}\right)}\left(\nabla^{2} \mathbf{v}^{\ell}, \nabla \pi^{\ell}\right)(t)\right\|_{L^{r}} \\
& \leq C\left\|\sigma^{\left(\frac{3}{2}-\frac{1}{r}-\frac{s_{\ell}}{2}\right)} D_{t} \mathrm{v}^{\ell}(t)\right\|_{L^{r}}+\mathcal{H}_{\ell-1}(t)\left(1+\left\|\nabla \mathrm{X}^{\ell-1}(t)\right\|_{W^{1, p}}\right) .
\end{aligned}
$$

Lemma 1.5 (Estimates for $g_{\ell}$ ). Under the same hypotheses in Lemma 1.3, one has

$$
\begin{aligned}
& \left\|g_{\ell}(t)\right\|_{L^{2}}+\sigma(t)^{\frac{1-s_{\ell-1}}{2}}\left\|\nabla g_{\ell}(t)\right\|_{L^{2}} \leq \mathcal{H}_{\ell-1}(t)\left(1+\left\|\nabla X^{\ell-1}(t)\right\|_{W^{1, p}}\right) \\
& \left\|\partial_{t} g_{\ell}(t)\right\|_{L^{2}} \leq\|v\|_{L^{\infty}}\left\|\nabla \mathbf{v}^{\ell}\right\|_{L^{2}}+\mathcal{H}_{\ell-1}(t) \sigma(t)^{-\left(1-\frac{s_{\ell-1}}{2}\right)}\left(1+\left\|\nabla X^{\ell-1}(t)\right\|_{W^{1, p}}\right) .
\end{aligned}
$$

1.4.3 $H^{1}$ energy estimate of $\mathrm{v}^{\ell}$

In this step we derive the time-weighted $H^{1}$ estimate of $\mathrm{v}^{\ell}$. We first decompose $\left(\mathrm{v}^{\ell}, \nabla \pi^{\ell}\right)$ as

$$
\mathrm{v}^{\ell}=\mathrm{v}_{\ell 1}+\mathrm{v}_{\ell 2} \quad \text { and } \quad \nabla \pi^{\ell}=\nabla \mathrm{p}_{\ell 1}+\nabla \mathrm{p}_{\ell 2}
$$

where $\left(\mathrm{v}_{\ell 1}, \nabla \mathrm{p}_{\ell 1}\right)$ solves the linear system (1.21) with the data $(f, g)=(0,0)$ :

$$
\left\{\begin{array}{l}
\rho \partial_{t} \mathrm{v}_{\ell 1}+\rho v \cdot \nabla \mathrm{v}_{\ell 1}-\Delta \mathrm{v}_{\ell 1}+\nabla \mathrm{p}_{\ell 1}=0 \\
\operatorname{div} \mathrm{v}_{\ell 1}=0 \\
\left.\mathrm{v}_{\ell 1}\right|_{t=0}=\partial_{X_{0}}^{\ell} v_{0}
\end{array}\right.
$$

and $\left(\mathrm{v}_{\ell 2}, \nabla \mathrm{p}_{\ell 2}\right)$ solves the linear system (1.21) with zero-initial data:

$$
\left\{\begin{array}{l}
\rho \partial_{t} \mathrm{v}_{\ell 2}+\rho v \cdot \nabla \mathrm{v}_{\ell 2}-\Delta \mathrm{v}_{\ell 2}+\nabla \mathrm{p}_{\ell 2}=f_{\ell}(v, \pi), \\
\operatorname{div}_{\ell 2}=\operatorname{div} g_{\ell,} \\
\left.\mathrm{v}_{\ell 2}\right|_{t=0}=0 .
\end{array}\right.
$$

It follows the Besov estimate and $H^{1}$ estimate for $\mathrm{v}_{\ell 1}$ directly from Theorem 1.1 (noticing that $(v, \nabla \pi)$ and $\left(\mathrm{v}_{\ell 1}, \nabla \mathrm{p}_{\ell 1}\right)$ both satisfy the linear system (1.21) with data $(f, g)=$ $(0,0))$ :

$$
\begin{aligned}
& \left\|\mathrm{v}_{\ell 1}\right\|_{\widetilde{L}_{t}^{\infty}\left(\dot{B}_{2,1}^{s_{\ell}}\right)}+\left\|\nabla \mathrm{v}_{\ell 1}\right\|_{\widetilde{L}_{t}^{2}\left(\dot{B}_{2,1}^{s_{\ell}}\right)}+\left\|\sigma^{\frac{1-s_{\ell}}{2}} \nabla \mathrm{v}_{\ell 1}\right\|_{L_{t}^{\infty}\left(L^{2}\right)}+\left\|\sigma^{\frac{1-s_{\ell}}{2}}\left(\partial_{t} \mathrm{v}_{\ell 1}, \nabla^{2} \mathrm{v}_{\ell 1}, \nabla \mathrm{p}_{\ell 1}\right)\right\|_{L_{t}^{2}\left(L^{2}\right)} \\
&
\end{aligned}
$$


Then we apply Lemma 1.1 to bound the time-weighted $H^{1}$-norm of $\mathrm{v}_{\ell 2}$ by

$$
\mathfrak{C}_{\ell}(X):=\mathcal{C}_{\ell} \exp \left(\mathcal{C}_{0}\langle t\rangle^{2}\right)+\mathcal{H}_{\ell-1}(t)\left(1+\int_{0}^{t} \sigma^{-\left(1-\frac{\theta_{0}}{2}\right)}\left\|\nabla X^{\ell-1}\left(t^{\prime}\right)\right\|_{W^{1, p}}^{2} d t^{\prime}\right),
$$

where $\theta_{0}=s_{\ell}-s_{\ell-1}$ be some constant in $(0,1)$. Hence we can bound $A_{\ell 1}^{2}$ by $\mathfrak{C}_{\ell}(X)$ :

Proposition 1.1 (The bound for $A_{\ell 1}$ ). Let $A_{\ell 1}(t)$ be given by (1.14a). Let $\mathfrak{C}_{\ell}(X)$ be given by (1.50). Under the assumptions of Lemma 1.3, we have

$$
A_{\ell 1}^{2}(t) \leq \mathfrak{C}_{\ell}(X) .
$$

We also get some consequent estimates from the bound $A_{\ell 1}^{2} \leq \mathfrak{C}_{\ell}(X)$ which will be useful in getting the $H^{1}$ estimate for $D_{t} \mathrm{v}_{\ell}$ :

Corollary 1.1 (Preliminary estimates for $D_{t} \mathrm{v}^{\ell}$ ). Under the assumptions of Proposition 1.1, one has

$$
\begin{aligned}
& \left\|\sigma^{\frac{1-s_{\ell}}{p}} \mathrm{v}^{\ell}\right\|_{L_{t}^{\infty}\left(L^{\frac{2 p}{p-2}}\right)}^{2}+\left\|\sigma^{\frac{1-s_{\ell}}{2}}\left(D_{t} \mathrm{v}^{\ell}, \nabla \partial_{X}^{\ell} \nabla v, D_{t} \partial_{X}^{\ell-1} \Delta X\right)\right\|_{L_{t}^{2}\left(L^{2}\right)}^{2} \\
& +\left\|\sigma^{\frac{1-s_{\ell}}{p}}\left(\nabla \mathbf{v}^{\ell}, \partial_{X}^{\ell} \nabla v, D_{t} \partial_{X}^{\ell-1} \nabla X\right)\right\|_{L_{t}^{2}\left(L^{\frac{2 p}{p-2}}\right)}^{2} \leq \mathfrak{C}_{\ell}(X) .
\end{aligned}
$$

\subsubsection{Estimates for $\left(\mathrm{F}_{\ell}, \mathfrak{a}_{\ell}, \mathfrak{b}_{\ell}\right)$}

We now get the estimates for the $\ell$-th data $\left(\mathrm{F}_{\ell}, \mathfrak{a}_{\ell}, \mathfrak{b}_{\ell}\right)$ defined in Subsection 1.2.3.

Lemma 1.6 (Estimate for $\mathrm{F}_{\ell}$ ). Under the assumptions of Lemma 1.3, we have the following estimates for $\mathrm{F}_{\ell}, \ell=2, \cdots, k$ :

$$
\left\|\sigma^{\frac{3-s_{\ell}}{2}} \mathrm{~F}_{\ell}\right\|_{L_{t}^{2}\left(L^{2}\right)}^{2} \leq \mathcal{H}_{\ell-1}^{2}(t)\left(\mathcal{C}_{\ell}+\int_{0}^{t}\left(B_{0}\left(t^{\prime}\right)+\sigma\left(t^{\prime}\right)^{-\left(1-\frac{\theta_{0}}{2}\right)}\right)\left\|\nabla \mathrm{X}^{\ell-1}\left(t^{\prime}\right)\right\|_{W^{1, p}}^{2} \mathrm{~d} t^{\prime}\right),
$$

for $B_{0}(t)$ given by $(1.17 \mathrm{~b})$.

Lemma 1.7 (Estimate for $\mathfrak{a}_{\ell}$ ). Under the hypotheses of Lemma 1.6, we have the following estimates for $\mathfrak{a}_{\ell}, \ell=2, \cdots, k$,

$$
\left\|\sigma^{\frac{1-s_{\ell}}{2}} \mathfrak{a}_{\ell}\right\|_{L_{t}^{2}\left(L^{2}\right)}^{2}+\left\|\sigma^{1-\frac{s_{\ell}}{2}} \nabla \mathfrak{a}_{\ell}\right\|_{L_{t}^{2}\left(L^{2}\right)}^{2}+\left\|\sigma^{\frac{3-s_{\ell}}{2}} \nabla \operatorname{div} \mathfrak{a}_{\ell}\right\|_{L_{t}^{2}\left(L^{2}\right)}^{2} \leq \widetilde{\mathfrak{C}}_{\ell}(X) .
$$

Here and in all that follows, for $\mathfrak{B}_{\ell}(t)$ determined by (1.43), we always designate

$$
\widetilde{\mathfrak{C}}_{\ell}(X):=\mathcal{H}_{\ell-1}^{2}(t)\left(\mathcal{C}_{\ell}+\int_{0}^{t}\left(\mathfrak{B}_{\ell-1}\left(t^{\prime}\right)+\sigma\left(t^{\prime}\right)^{-\left(1-\frac{\theta_{0}}{2}\right)}\right)\left\|\nabla X^{\ell-1}\left(t^{\prime}\right)\right\|_{W^{1, p}}^{2} \mathrm{~d} t^{\prime}\right) .
$$

Lemma 1.8 (Estimate for $\mathfrak{b}_{\ell}$ ). Under the hypotheses of Lemma 1.7, for any $\varepsilon>0$, there exists $a$ constant $C_{\varepsilon}$ such that for $\mathfrak{b}_{\ell}, \ell=2, \cdots, k$ we have

$$
\left\|\sigma^{\frac{3-s_{\ell}}{2}} \mathfrak{b}_{\ell}\right\|_{L_{t}^{2}\left(L^{2}\right)}^{2} \leq C \varepsilon\left\|\sigma^{\frac{3-s_{\ell}}{2}} D_{t}^{2} \mathbf{v}^{\ell}\right\|_{L_{t}^{2}\left(L^{2}\right)}^{2}+C_{\varepsilon} \widetilde{\mathfrak{C}}_{\ell}(X),
$$

with $\widetilde{\mathfrak{C}}_{\ell}(X)$ given by (1.54). 


\subsubsection{Proof of (1.19)}

We apply Lemma 1.2 and the estimates for the data in Lemmas 1.6-1.7-1.8 to the equation (1.32) to get the $H^{1}$-estimate for $D_{t} \mathrm{v}^{\ell}$, which will be used to get the $W^{2, p}$-estimate for $\mathrm{X}^{\ell-1}$ :

Proposition 1.2 (Bounds for $A_{\ell 1}, A_{\ell 2}, \mathrm{X}^{\ell-1}$ ). Let $A_{\ell 1}(t), A_{\ell 2}(t)$ be given by (1.14a). Then under the assumptions of Lemma 1.6, we have

$$
A_{\ell 1}(t)+A_{\ell 2}(t)+\left\|\mathrm{X}^{\ell-1}\right\|_{L_{t}^{\infty}\left(W^{2, p}\right)} \leq \mathcal{H}_{\ell}(t)
$$

With Proposition 1.2 in hand, to complete the proof of the bound (1.19) $A_{\ell}(t) \leq \mathcal{H}_{\ell}(t)$, it remains to show the $\dot{B}_{2,1}^{s_{\ell}}$ estimate for $\mathrm{v}^{\ell}$ :

$$
\left\|\mathrm{v}^{\ell}\right\|_{\widetilde{L}_{t}^{\infty}\left(\dot{B}_{2,1}^{s_{\ell}}\right)}+\left\|\nabla \mathrm{v}^{\ell}\right\|_{\widetilde{L}_{t}^{2}\left(\dot{B}_{2,1}^{s_{\ell}}\right)} \leq \mathcal{H}_{\ell}(t)
$$

The proof of (1.57) follows exactly the same argument as those in proofs of the Besov estimates in Theorem 1.1 (see [26]). It is delicate but does not contain substantial new ideas. We skip the details here.

\section{Proofs}

We follow the procedure in Subsection 1.4 to prove the estimate (1.19) $A_{\ell}(t) \leq \mathcal{H}_{\ell}(t)$ from the inductive assumption (1.37) $A_{l}(t) \leq \mathcal{H}_{l}(t), \forall l \leq \ell-1$.

\subsection{Deductive estimates from the inductive assumption}

In this subsection we shall derive more estimates (1.38) from the inductive assumption (1.37), which will be used constantly in the following context.

First of all, we state the lemma concerning the commutator estimates:

Lemma 2.1 (Commutator estimates). Let $\ell \in\{1, \cdots, k\}$ and $(i, j)$ be any pair of nonnegative integers with $i+j \leq \ell$. Then for $r_{1}, r_{,} r_{3}$ satisfying (1.40), there exists a positive constant $C$ such that

$$
\begin{aligned}
& \left\|\partial_{X}^{i} \nabla X^{\ell-i}-\nabla X^{\ell}\right\|_{L_{t}^{\infty}\left(W^{1, p}\right)}+\left\|\partial_{X}^{i} \nabla \partial_{X}^{j} \nabla X^{\ell-i-j}-\nabla^{2} X^{\ell}\right\|_{L_{t}^{\infty}\left(L^{p}\right)} \leq C R_{\ell}^{2}(t) \\
& \left\|\sigma^{\left(1-\frac{1}{r_{1}}-\frac{s_{\ell-1}}{2}\right)}\left(\partial_{X}^{i} \nabla \mathbf{v}^{\ell-i}-\nabla \mathbf{v}^{\ell}\right)\right\|_{L_{t}^{\infty}\left(L^{r_{1}}\right)}+\left\|\sigma^{\left(\frac{3}{2}-\frac{1}{r}-\frac{s_{\ell-1}}{2}\right)}\left(\partial_{X}^{i} \nabla \pi^{\ell-i}-\nabla \pi^{\ell}\right)\right\|_{L_{t}^{\infty}\left(L^{r}\right)} \\
& \quad+\left\|\sigma^{\left(\frac{3}{2}-\frac{1}{r}-\frac{s_{\ell-1}}{2}\right)}\left(\partial_{X}^{i} \nabla \partial_{X}^{j} \nabla \mathbf{v}^{\ell-i-j}-\nabla^{2} \mathbf{v}^{\ell}\right)\right\|_{L_{t}^{\infty}\left(L^{r}\right)} \leq C R_{\ell}(t) \mathfrak{A}_{\ell-1}(t)
\end{aligned}
$$


and

$$
\begin{aligned}
& \left\|\sigma(t)^{\left(\frac{3}{2}-\frac{1}{r_{3}}-\frac{s_{\ell-1}}{2}\right)}\left(\partial_{X}^{i} \nabla D_{t} \mathrm{v}^{\ell-i}-\nabla D_{t} \mathrm{v}^{\ell}, \partial_{X}^{i} D_{t} \nabla \mathrm{v}^{\ell-i}-\nabla D_{t} \mathrm{v}^{\ell}\right)(t)\right\|_{L^{r_{3}}}^{2} \\
& +\left\|\sigma(t)^{\frac{3-s_{\ell-1}}{2}}\left(\partial_{X}^{i} D_{t} \nabla^{2} \mathrm{v}^{\ell-i}-\nabla^{2} D_{t} \mathrm{v}^{\ell}, \nabla \partial_{X}^{i} \nabla D_{t} \mathrm{v}^{\ell-i}-\nabla^{2} D_{t} \mathrm{v}^{\ell}\right)(t)\right\|_{L^{2}}^{2} \\
& +\left\|\sigma(t)^{\frac{3-s_{\ell-1}}{2}}\left(\partial_{X}^{i} D_{t} \nabla \pi^{\ell-i}-\nabla D_{t} \pi^{\ell}\right)(t)\right\|_{L^{2}}^{2} \leq C R_{\ell}^{2}(t) \mathfrak{B}_{\ell-1}(t)+C \mathfrak{A}_{\ell}^{4}(t) \sigma^{-\left(1-s_{\ell}\right) .}
\end{aligned}
$$

This lemma follows from the same argument as that in the proof of Lemma 4.1 of [25]. It is elementary and we skip the details. Interested readers are referred to Appendix A of [27] for details.

We derive (1.38) for $\ell=1$ immediately from Theorem 1.1 and the proof can be found in Section 5.2 [27]:

Proposition 2.1. Under the hypotheses of Theorem 1.1, we have (1.38) for $l=1$ :

$$
\|\nabla X\|_{L_{t}^{\infty}\left(W^{1, p}\right)}+\mathfrak{A}_{1}(t)+\int_{0}^{t} \mathfrak{B}_{1}\left(t^{\prime}\right) d t^{\prime} \leq \mathcal{H}_{1}(t) .
$$

By view of (2.3) we can derive more general inductive assumptions (1.38) in Lemma 1.3 by an inductive argument.

Proof of Lemma 1.3. By (2.3), we can assume that

$$
R_{\mathcal{K}}(t)+\mathfrak{A}_{\mathcal{K}}(t)+\int_{0}^{t} \mathfrak{B}_{\mathcal{K}}\left(t^{\prime}\right) d t^{\prime} \leq \mathcal{H}_{\mathcal{K}}(t), \quad 1 \leq \kappa \leq l-1 .
$$

We shall show the estimate (2.4) for $\kappa+1$. Indeed, it follows from (1.37) that

$$
\left\|\nabla X^{\beta}\right\|_{L_{t}^{\infty}\left(W^{1, p}\right)} \leq \mathcal{H}_{\beta+1}(t), \quad \forall \beta \leq l-1,
$$

which together with the commutator estimate (2.1) for $\kappa$ and (2.4) ensures that

$$
R_{\kappa+1}(t) \leq \mathcal{H}_{\kappa+1}(t)
$$

Similarly, due to the estimate (2.5) and the commutator estimates (2.1b)-(2.2a), we can prove (2.4) for $\kappa+1$ from the assumption (1.37). We skip the details and interested readers may check the proof of Lemma 6.2 in [27] for details.

\subsection{Estimates for $\left(f_{\ell}, g_{\ell}\right)$}

In this subsection we prove the estimates (1.44)-(1.45a) in Lemmas 1.4-1.5 for the data $\left(f_{\ell}, g_{\ell}\right)$ defined in Subsection 1.2.3 respectively, by use of the general inductive assumption (1.38). 
Proof of Lemma 1.4. Recall the definition of $f_{\ell}$ in (1.29). For $r=2$ or $p$, we deduce

$$
\begin{aligned}
\left\|f_{\ell}\right\|_{L^{r}} \leq C & \left(R_{\ell-1}(t)+\left\|\partial_{X}^{\ell-1} \nabla X\right\|_{L^{\infty}}+\left\|\partial_{X}^{\ell-1} \Delta X\right\|_{L^{p}}\right) \\
& \times \sum_{i+j \leq \ell-1}\left(\left\|\left(D_{t} \mathrm{v}^{j}, \partial_{X}^{i} \nabla^{2} \mathrm{v}^{j}, \partial_{X}^{i} \nabla \pi^{j}\right)\right\|_{L^{r}}+\left\|\partial_{X}^{i} \nabla \mathrm{v}^{j}\right\|_{L^{\frac{p r}{p-r}}}\right),
\end{aligned}
$$

which together with (2.1) ensures that for $r=2$ or $r=p$

$$
\left\|f_{\ell}\right\|_{L^{r}} \leq C\left(R_{\ell-1}(t)+R_{\ell-1}^{2}(t)+\left\|\nabla X^{\ell-1}\right\|_{W^{1, p}}\right) \sigma^{-\left(\frac{3}{2}-\frac{1}{r}-\frac{s_{\ell-1}}{2}\right)} \mathfrak{A}_{\ell-1}(t) .
$$

As a result, we deduce from (1.38) that for $r=2$ or $r=p$

$$
\left\|f_{\ell}\right\|_{L^{r}} \leq \mathcal{H}_{\ell-1}(t) \sigma(t)^{-\left(\frac{3}{2}-\frac{1}{r}-\frac{s_{\ell-1}}{2}\right)}\left(1+\left\|\nabla X^{\ell-1}\right\|_{W^{1, p}}\right) .
$$

Recall in Subsection 1.2.3 that

$$
\begin{aligned}
\operatorname{divv}^{\ell} & =\operatorname{div} g_{\ell}=\sum_{i=0}^{\ell-1} \operatorname{div} P_{X}^{i}\left(\mathrm{v}^{\ell-1-i} \cdot \nabla X\right)=\sum_{i=0}^{\ell-1} \partial_{X}^{i} \operatorname{div}\left(\mathrm{v}^{\ell-1-i} \cdot \nabla X\right) \\
& =\sum_{i=0}^{\ell-1} \partial_{X}^{i}\left(\partial_{\alpha} \mathrm{v}^{\ell-1-i} \cdot \nabla X^{\alpha}\right)
\end{aligned}
$$

and hence

$$
\nabla \operatorname{div} \mathrm{v}^{\ell}=\sum_{i=0}^{\ell-1} \sum_{j=0}^{i} C_{i}^{j} \nabla\left(\partial_{X}^{j} \partial_{\alpha} \mathrm{v}^{\ell-1-i} \cdot \partial_{X}^{i-j} \nabla X^{\alpha}\right),
$$

from which, we infer for $r=2$ or $r=p$

$$
\begin{aligned}
\left\|\nabla \operatorname{divv}^{\ell}\right\|_{L^{r}} \leq C & \left(R_{\ell-1}(t)+\left\|\partial_{X}^{\ell-1} \nabla X\right\|_{L^{\infty}}+\left\|\nabla \partial_{X}^{\ell-1} \nabla X\right\|_{L^{p}}\right) \\
& \times \sum_{i+j \leq \ell-1}\left(\left\|\nabla \partial_{X}^{i} \nabla \mathrm{v}^{j}\right\|_{L^{r}}+\left\|\partial_{X}^{i} \nabla \mathrm{v}^{j}\right\|_{L^{\frac{p r}{p-r}}}\right) .
\end{aligned}
$$

Then we deduce from the commutator estimate (2.1) that for $r=2, p$,

$$
\left.\left\|\nabla \operatorname{divv}^{\ell}\right\|_{L^{r}} \leq C\left(R_{\ell-1}(t)+R_{\ell-1}^{2}(t)+\left\|\nabla X^{\ell-1}\right\|_{W^{1, p}}\right) \sigma^{-\left(\frac{3}{2}-\frac{1}{r}-\frac{s_{\ell-1}}{2}\right.}\right)_{\mathfrak{A}_{\ell-1}}(t) .
$$

By virtue of (1.38) and (2.7), we obtain the first inequality of (1.44).

On the other hand, we rewrite the $\mathrm{v}^{\ell}$-equation (1.29) as

$$
-\Delta\left(\mathrm{v}^{\ell}-\nabla \Delta^{-1} \operatorname{div}^{\ell}\right)+\nabla \pi^{\ell}=\nabla \operatorname{div} \mathrm{v}^{\ell}-\rho D_{t} \mathrm{v}^{\ell}+f_{\ell}(v, \pi),
$$

so that for any $r \in(1, \infty)$, it follows from classical estimates for Stokes operator that

$$
\left\|\left(\nabla^{2} \mathrm{v}^{\ell}, \nabla \pi^{\ell}\right)\right\|_{L^{r}} \leq C\left(\left\|\nabla \operatorname{divv}^{\ell}\right\|_{L^{r}}+\left\|\rho D_{t} \mathrm{v}^{\ell}\right\|_{L^{r}}+\left\|f_{\ell}\right\|_{L^{r}}\right),
$$

which together with the first inequality of (1.44) gives rise to the second one of (1.44). 
Proof of Lemma 1.5. Recall the definition of $g_{\ell}$ in Subsection 1.2.3:

$$
\begin{aligned}
g_{\ell} & =\sum_{i=0}^{\ell-1}\left(\partial_{X}-(\nabla X)^{T} \cdot\right)^{i}\left(\mathrm{v}^{\ell-1-i} \cdot \nabla X\right) \\
& =\sum_{i=0}^{\ell-1} \sum_{i_{1}+\cdots+i_{r}=i} \partial_{X}^{i_{1}}\left(\mathrm{v}^{\ell-1-i} \cdot \nabla X\right) \cdot \partial_{X}^{i_{2}}(-\nabla X)^{i_{3}} \cdots \partial_{X}^{i_{r-1}}(-\nabla X)^{i_{r}} .
\end{aligned}
$$

It is obvious to observe from Lemmas 2.1 and 1.3 that

$$
\begin{aligned}
\left\|g_{\ell}\right\|_{L^{2}} & \leq\left\|v \cdot \partial_{X}^{\ell-1} \nabla X\right\|_{L^{2}}+\sum_{l \leq \ell-2, i \leq \ell-1}\left\|\mathrm{v}^{i}\right\|_{L^{2}}\left\|\partial_{X}^{l} \nabla X\right\|_{L^{\infty}}^{\ell-1} \\
& \leq\left(\sum_{i \leq \ell-1}\left\|\mathrm{v}^{i}\right\|_{L^{2}}\right)\left(\left\|\partial_{X}^{\ell-1} \nabla X\right\|_{L^{\infty}}+R_{\ell-1}^{\ell-1}(t)\right) \\
& \leq \mathcal{H}_{\ell-1}(t)\left(1+\left\|\nabla X^{\ell-1}\right\|_{W^{1, p}}\right) .
\end{aligned}
$$

While by taking $\partial_{\kappa}$ with $\kappa=0,1,2$ (here $\partial_{0} \stackrel{\text { def }}{=} \partial_{t}$ ) to (2.10), we write

$$
\partial_{\kappa} g_{\ell}=g_{\ell, \kappa}^{1}+g_{\ell, \kappa}^{2}
$$

where

$$
\begin{aligned}
g_{\ell, \kappa}^{1}:= & \sum_{i=1}^{\ell-1} \sum_{i_{1}+\cdots+i_{r}=i-1}\left(\partial_{X}^{i_{1}}\left(\partial_{\mathcal{K}} X \cdot \nabla \partial_{X}^{i_{2}}\left(\mathrm{v}^{\ell-1-i} \cdot \nabla X\right)\right) \cdots \partial_{X}^{i_{r-1}}(-\nabla X)^{i_{r}}\right. \\
& +\partial_{X}^{i_{1}}\left(\mathrm{v}^{\ell-1-i} \cdot \nabla X\right) \cdots \partial_{X}^{i_{j-1}}\left(\partial_{\kappa} X \cdot \nabla \partial_{X}^{i_{j}}(-\nabla X)^{i_{j+1}}\right) \cdots \partial_{X}^{i_{r-1}}(-\nabla X)^{i_{r}} \\
& \left.+\partial_{X}^{i_{1}}\left(\mathrm{v}^{\ell-1-i} \cdot \nabla X\right) \cdot \partial_{X}^{i_{2}}(-\nabla X)^{i_{3}} \cdots \partial_{X}^{i_{j}} \partial_{\kappa}(-\nabla X) \cdots \partial_{X}^{i_{r-1}}(-\nabla X)^{i_{r}}\right),
\end{aligned}
$$

and

$$
g_{\ell, \kappa}^{2}:=\sum_{i=0}^{\ell-1} \sum_{i_{1}+\cdots+i_{r}=i} \partial_{X}^{i_{1}} \partial_{\kappa}\left(\mathrm{v}^{\ell-1-i} \cdot \nabla X\right) \cdot \partial_{X}^{i_{2}}(-\nabla X)^{i_{3}} \cdots \partial_{X}^{i_{r-1}}(-\nabla X)^{i_{r}} .
$$

Notice that the indices $i_{1}, i_{2}, \cdots, i_{r}$ satisfy $i_{1}+\cdots i_{r} \leq \ell-2$ in $g_{\ell, \kappa}^{1}$. We thus get from (1.38) that for $\kappa=1,2$,

$$
\left\|g_{\ell, \kappa}^{1}\right\|_{L^{2}} \leq C \sum_{i+j \leq \ell-2} \mathcal{H}_{\ell-1}(t)\left(\left\|\partial_{X}^{i} \nabla \mathbf{v}^{j}\right\|_{L^{2}}+\left\|\mathbf{v}^{i}\right\|_{L^{\frac{2 p}{p-2}}}\right) \leq C \mathcal{H}_{\ell-1}(t) \sigma^{-\frac{1-s_{\ell-1}}{2}} \mathfrak{A}_{\ell-1}(t) .
$$

To handle $g_{\ell, \kappa}^{2}$, we separate the case when $i_{1}=i=\ell-1$ from others to get

$$
\begin{aligned}
\left\|g_{\ell, \kappa}^{2}\right\|_{L^{2}} \leq\|\nabla v\|_{L^{2}}\left\|\partial_{X}^{\ell-1} \nabla X\right\|_{L^{\infty}}+\|v\|_{L^{\frac{2 p}{p-2}}}\left\|\partial_{X}^{\ell-1} \nabla^{2} X\right\|_{L^{p}} \\
+C \sum_{i+j \leq \ell-1} \mathcal{H}_{\ell-1}(t)\left(\left\|\partial_{X}^{i} \nabla \mathrm{v}^{j}\right\|_{L^{2}}+\left\|\mathrm{v}^{i}\right\|_{L^{\frac{2 p}{p-2}}}\right),
\end{aligned}
$$


from which and the commutator estimate (2.1), we infer

$$
\left\|g_{\ell, \kappa}^{2}\right\|_{L^{2}} \leq C\left(\mathcal{H}_{\ell-1}(t)+\left\|\nabla X^{\ell-1}\right\|_{W^{1, p}}\right) \sigma^{-\frac{1-s_{\ell-1}}{2}} \mathfrak{A}_{\ell-1}(t) .
$$

Combining the above estimates of $g_{\ell, \kappa}^{1}$ and $g_{\ell, \kappa}^{2}, \kappa=1,2$ with Lemma 1.3, we obtain the first estimate of (1.45a).

Along the same line, we deduce from (2.12) that

$$
\begin{gathered}
\left\|g_{\ell, 0}^{1}\right\|_{L^{2}} \leq \mathcal{H}_{\ell-1}(t) \sigma^{-\left(1-\frac{s_{\ell-1}}{2}\right)} \sum_{\substack{i+j \leq \ell-2 \\
l \leq \ell-2}}\left(\| \sigma ^ { \frac { 1 } { 2 } } \partial _ { X } ^ { l } \partial _ { t } X \| _ { L ^ { \infty } } \left(\left\|\sigma^{\frac{1-s_{\ell-1}}{2}} \partial_{X}^{i} \nabla \mathrm{v}^{j}\right\|_{L^{2}}\right.\right. \\
\left.\left.+\left\|\sigma^{\frac{1-s_{\ell-1}}{2}} \mathrm{v}^{i}\right\|_{L^{\frac{2 p}{p-2}}}\right)+\left\|\sigma^{\frac{1-s_{\ell-1}}{2}} \partial_{X}^{l} \nabla \partial_{t} X\right\|_{L^{2}}\left\|\sigma^{\frac{1}{2}} \mathrm{v}^{i}\right\|_{L^{\infty}}\right)
\end{gathered}
$$

from which and Lemma 1.3, we infer that

$$
\left\|g_{\ell, 0}^{1}\right\|_{L^{2}} \leq \mathcal{H}_{\ell-1}(t) \sigma^{-\left(1-\frac{s_{\ell-1}}{2}\right)} \mathfrak{A}_{\ell-1}^{2}(t) \leq \mathcal{H}_{\ell-1}(t) \sigma^{-\left(1-\frac{s_{\ell-1}}{2}\right)} .
$$

While by separating the case when $i_{1}=i=\ell-1$ from others and taking into account of the fact that: $\partial_{t} X=-v \cdot \nabla X+v^{1}$ one has

$$
\begin{aligned}
\left\|g_{\ell, 0}^{2}\right\|_{L^{2}} \leq & \left\|\partial_{t} v \cdot \partial_{X}^{\ell-1} \nabla X\right\|_{L^{2}}+\left\|v \otimes \partial_{X}^{\ell-1} \nabla(v \cdot \nabla X)\right\|_{L^{2}}+\left\|v \cdot \partial_{X}^{\ell-1} \nabla \mathrm{v}^{1}\right\|_{L^{2}} \\
& +C \sum_{\substack{i+j \leq \ell-1 \\
l+m \leq \ell-1, m \leq \ell-2}} \mathcal{H}_{\ell-1}(t)\left(\left\|\partial_{X}^{i} \partial_{t} \mathrm{v}^{j}\right\|_{L^{2}}+\left\|\mathrm{v}^{l}\right\|_{L^{\infty}}\left\|\partial_{X}^{m} \nabla \partial_{t} X\right\|_{L^{2}}\right),
\end{aligned}
$$

which yields

$$
\begin{aligned}
\left\|g_{\ell, 0}^{2}\right\|_{L^{2}} \leq & \left(\left\|\left(\partial_{t} v, v \otimes \nabla v\right)\right\|_{L^{2}}+\|v \otimes v\|_{L^{\frac{2 p}{p-2}}}\right)\left(\left\|\partial_{X}^{\ell-1} \nabla X\right\|_{L^{\infty}}+\left\|\partial_{X}^{\ell-1} \nabla^{2} X\right\|_{L^{p}}\right) \\
& +\left\|v \cdot \nabla \mathbf{v}^{\ell}\right\|_{L^{2}}+\sum_{i+j \leq \ell-1}\left(\left\|v \otimes \partial_{X}^{i} \nabla v^{j}\right\|_{L^{2}}+\left\|v \otimes v^{i}\right\|_{L^{\frac{2 p}{p-2}}}\right) R_{\ell-1}(t) \\
& +C \mathcal{H}_{\ell-1}(t) \sigma^{-\left(1-\frac{s_{\ell-1}}{2}\right)}\left(\mathfrak{A}_{\ell-1}(t)+\mathfrak{A}_{\ell-1}^{2}(t)\right),
\end{aligned}
$$

that is

$$
\left\|g_{\ell, 0}^{2}\right\|_{L^{2}} \leq\|v\|_{L^{\infty}}\left\|\nabla \mathbf{v}^{\ell}\right\|_{L^{2}}+\mathcal{H}_{\ell-1}(t) \sigma^{-\left(1-\frac{s_{\ell-1}}{2}\right)}\left(1+\left\|\nabla \mathbf{X}^{\ell-1}\right\|_{W^{1, p}}\right) .
$$

The above estimates for $g_{\ell, 0^{\prime}}^{1} g_{\ell, 0}^{2}$ imply the second estimate of (1.45a).

\section{3 $\quad H^{1}$ energy estimate of $\mathrm{v}^{\ell}$}

In this subsection, we prove the time-weighted $H^{1}$ estimate of $\mathrm{v}^{\ell}$ in Proposition 1.1 and the consequent estimates for $D_{t} \mathrm{v}^{\ell}$ in Corollary 1.1. 
Proof of Proposition 1.1. We first apply Lemma 1.1 to the $\mathrm{v}_{\ell 2}$-equation (1.48) that

$$
\begin{gathered}
\left\|\sigma^{\frac{1-s_{\ell}}{2}} \nabla \mathrm{v}_{\ell 2}\right\|_{L_{t}^{\infty}\left(L^{2}\right)}^{2}+\left\|\sigma^{\frac{1-s_{\ell}}{2}}\left(\partial_{t} \mathrm{v}_{\ell 2}, \nabla^{2} \mathrm{v}_{\ell 2}, \nabla \mathrm{p}_{\ell 2}\right)\right\|_{L_{t}^{2}\left(L^{2}\right)} \\
\leq \mathcal{C}_{0}\langle t\rangle\left(\left\|g_{\ell}(0)\right\|_{\dot{B}_{2,1}^{s_{\ell-1}}}^{2}+\left\|\sigma^{-\frac{s_{\ell}}{2}}\left(\partial_{t} g_{\ell}, f_{\ell}\right)\right\|_{L_{t}^{1}\left(L^{2}\right)}^{2}+\left\|\sigma^{-\frac{1-\left(s_{0}-s_{\ell}\right)}{2}} \nabla g_{\ell}\right\|_{L_{t}^{1}\left(L^{2}\right)}^{2}\right. \\
\left.+\left\|\sigma^{-\frac{s_{\ell}}{2}} \nabla g_{\ell}\right\|_{L_{t}^{2}\left(L^{2}\right)}^{2}+\left\|\sigma^{\frac{1-s_{\ell}}{2}}\left(\partial_{t} g_{\ell}, \nabla \operatorname{div} g_{\ell}, f_{\ell}\right)\right\|_{L_{t}^{2}\left(L^{2}\right)}^{2}\right) .
\end{gathered}
$$

In view of the formulation (2.10) of $g_{\ell}$, we get, by applying the law of product in Besov spaces (see [5] for instance), that

$$
\begin{aligned}
& \left\|g_{\ell}(0)\right\|_{\dot{B}_{2,1}^{s} \ell-1} \leq C \sum_{i, j, l \leq \ell-1}\left\|\mathrm{v}^{i}(0)\right\|_{\dot{B}_{2,1}^{s_{\ell-1}}}\left\|\partial_{X_{0}}^{j} \nabla X_{0}\right\|_{\dot{B}_{p, 1}^{\frac{2}{p}}}^{l} \\
& \leq C \sum_{i, j \leq \ell-1}\left\|\mathrm{v}^{i}(0)\right\|_{\dot{B}_{2,1}^{s} \ell-1}\left\|\partial_{X_{0}}^{j} \nabla X_{0}\right\|_{W^{1, p}}^{l} \\
& \leq C\left(\|v\|_{L^{2} \cap \dot{B}_{2,1}^{s_{0}}}, \cdots,\left\|\partial_{X_{0}}^{\ell-1} v_{0}\right\|_{\dot{B}_{2,1}^{s_{\ell-1}}},\left\|X_{0}\right\|_{W^{2, p}, \cdots},\left\|\partial_{X_{0}}^{\ell-1} X_{0}\right\|_{W^{2, p}}\right) \leq \mathcal{C}_{\ell} .
\end{aligned}
$$

While it follows from Lemma 1.4 and $\theta_{0}=s_{\ell-1}-s_{\ell}$ that

$$
\begin{gathered}
\left\|\sigma^{-\frac{s_{\ell}}{2}} f_{\ell}\right\|_{L_{t}^{1}\left(L^{2}\right)}^{2}+\left\|\sigma^{\frac{1-s_{\ell}}{2}}\left(\nabla \operatorname{div} g_{\ell}, f_{\ell}\right)\right\|_{L_{t}^{2}\left(L^{2}\right)}^{2} \\
\leq \mathcal{H}_{\ell-1}^{2}(t) \int_{0}^{t} \sigma^{-\left(1-\frac{\theta_{0}}{2}\right)}\left(1+\left\|\nabla X^{\ell-1}\right\|_{W^{1, p}}^{2}\right) d t^{\prime} .
\end{gathered}
$$

And Lemma 1.5 ensures that

$$
\begin{aligned}
& \left\|\sigma^{-\frac{s_{\ell}}{2}} \partial_{t} g_{\ell}\right\|_{L_{t}^{1}\left(L^{2}\right)}^{2}+\left\|\sigma^{-\frac{1-\left(s_{0}-s_{\ell}\right)}{2}} \nabla g_{\ell}\right\|_{L_{t}^{1}\left(L^{2}\right)}^{2}+\left\|\sigma^{-\frac{s_{\ell}}{2}} \nabla g_{\ell}\right\|_{L_{t}^{2}\left(L^{2}\right)}^{2}+\left\|\sigma^{\frac{1-s_{\ell}}{2}} \partial_{t} g_{\ell}\right\|_{L_{t}^{2}\left(L^{2}\right)}^{2} \\
& \leq C\langle t\rangle\left\|\sigma^{\frac{1-s_{0}}{2}} v\right\|_{L_{t}^{\infty}\left(L^{\infty}\right)}^{2} \int_{0}^{t} \sigma^{-\left(1-\frac{s_{0}}{2}\right)}\left(\left\|\sigma^{\frac{1-s_{\ell}}{2}} \nabla \mathrm{v}_{\ell 1}\right\|_{L^{2}}^{2}+\left\|\sigma^{\frac{1-s_{\ell}}{2}} \nabla \mathrm{v}_{\ell 2}\right\|_{L^{2}}^{2}\right) \mathrm{d} t^{\prime} \\
& \quad+\mathcal{H}_{\ell-1}^{2}(t) \int_{0}^{t} \sigma^{-\left(1-\frac{\theta_{0}}{2}\right)}\left(1+\left\|\nabla X^{\ell-1}\right\|_{W^{1, p}}^{2}\right) \mathrm{d} t^{\prime}
\end{aligned}
$$

Inserting the above estimates together with the $H^{1}$ estimate (1.49) for $\mathrm{v}_{\ell 1}$ into (2.16) and applying Gronwall's inequality results in

$$
\left\|\sigma^{\frac{1-s_{\ell}}{2}} \nabla \mathrm{v}_{\ell 2}\right\|_{L_{t}^{\infty}\left(L^{2}\right)}^{2}+\left\|\sigma^{\frac{1-s_{\ell}}{2}}\left(\partial_{t} \mathrm{v}_{\ell 2}, \nabla^{2} \mathrm{v}_{\ell 2}, \nabla \mathbf{p}_{\ell 2}\right)\right\|_{L_{t}^{2}\left(L^{2}\right)}^{2} \leq \mathfrak{C}_{\ell}(X)
$$

for $\mathfrak{C}_{\ell}(X)$ given by (1.50). 
This above estimate implies the $L^{2}$ energy estimate of $v_{\ell 2}$. Indeed, we take $L^{2}\left(\mathbb{R}^{2}\right)$ inner product of (1.48) with $\mathrm{v}_{\ell 2}$ to get

$$
\begin{aligned}
\frac{1}{2} \frac{d}{d t}\left\|\sqrt{\rho} \mathbf{v}_{\ell 2}\right\|_{L^{2}}^{2}+\left\|\nabla \mathbf{v}_{\ell 2}\right\|_{L^{2}}^{2} & =\int_{\mathbb{R}^{2}}\left(f_{\ell}-\nabla \mathrm{p}_{\ell 2}\right) \cdot \mathbf{v}_{\ell 2} d x \\
& \leq\left\|\sigma^{\frac{1-s_{\ell}}{2}}\left(f_{\ell}, \nabla \mathbf{p}_{\ell 2}\right)\right\|_{L_{t}^{2}\left(L^{2}\right)}^{2}+\int_{0}^{t} \sigma^{-\left(1-s_{\ell}\right)}\left\|\mathrm{v}_{\ell 2}\right\|_{L^{2}}^{2} d x
\end{aligned}
$$

Hence we apply Gronwall's inequality to get

$$
\left\|\mathrm{v}_{\ell 2}\right\|_{L_{t}^{\infty}\left(L^{2}\right)}^{2}+\left\|\nabla \mathrm{v}_{\ell 2}\right\|_{L_{t}^{2}\left(L^{2}\right)}^{2} \leq \mathfrak{C}_{\ell}(X) .
$$

We sum up the above $H^{1}$ estimates for $\mathrm{v}_{\ell 2}$ and the $H^{1}$ estimate (1.49) for $\mathrm{v}_{\ell 1}$ to get $A_{\ell 1}^{2}(t) \leq$ $\mathfrak{C}_{\ell}(X)$.

Proof of Corollary 1.1. We will derive the estimates (1.52) from the bound (1.16) in Theorem 1.1, the bound $A_{\ell 1}^{2} \leq \mathfrak{C}_{\ell}(X)$, the commutator estimates in Lemma 2.1 and the inductive assumptions in Lemma 1.3.

We first deduce from the bound $A_{\ell 1}^{2} \leq \mathfrak{C}_{\ell}(X)$ and Gagliardo-Nirenberg's inequality that

$$
\left\|\sigma^{\frac{1-s_{\ell}}{p}} \mathrm{v}^{\ell}\right\|_{L_{t}^{\infty}\left(L^{\left.\frac{2 p}{p-2}\right)}\right.}^{2} \leq C\left(\left\|\mathrm{v}^{\ell}\right\|_{L_{t}^{\infty}\left(L^{2}\right)}^{1-\frac{2}{p}}\left\|\sigma^{\frac{1-s_{\ell}}{2}} \nabla \mathrm{v}^{\ell}\right\|_{L_{t}^{\infty}\left(L^{2}\right)}^{\frac{2}{p}}\right)^{2} \leq \mathfrak{C}_{\ell}(X),
$$

and

$$
\left\|\sigma^{\frac{1-s_{\ell}}{2}} D_{t} \mathbf{v}^{\ell}\right\|_{L_{t}^{2}\left(L^{2}\right)}^{2} \leq\left\|\sigma^{\frac{1-s_{\ell}}{2}} \partial_{t} \mathbf{v}^{\ell}\right\|_{L_{t}^{2}\left(L^{2}\right)}^{2}+\|v\|_{L_{t}^{2}\left(L^{\infty}\right)}^{2}\left\|\sigma^{\frac{1-s_{\ell}}{2}} \nabla \mathbf{v}^{\ell}\right\|_{L_{t}^{\infty}\left(L^{2}\right)}^{2} \leq \mathfrak{C}_{\ell}(X) .
$$

In order to estimate $\nabla \partial_{X}^{\ell} \nabla v$, we consider its difference from $\nabla^{2} \mathrm{v}^{\ell}$ :

$$
\nabla \partial_{X}^{\ell} \nabla v-\nabla^{2} \mathrm{v}^{\ell}=\nabla \sum_{i=0}^{\ell-1} \partial_{X}^{i}\left[\partial_{X} ; \nabla\right] \mathrm{v}^{\ell-1-i}=-\sum_{i=0}^{\ell-1} \nabla \partial_{X}^{i}\left(\nabla X \cdot \nabla \mathrm{v}^{\ell-1-i}\right) .
$$

Indeed, we know

$$
\begin{aligned}
& \left\|\sigma^{\frac{1-s_{\ell}}{2}}\left(\nabla \partial_{X}^{\ell} \nabla v-\nabla^{2} \mathbf{v}^{\ell}\right)\right\|_{L_{t}^{2}\left(L^{2}\right)}^{2} \\
& \lesssim\left(\left\|\sigma^{1-\frac{s_{0}}{2}} \nabla v\right\|_{L_{t}^{\infty}\left(L^{\frac{2 p}{p-2}}\right)}^{2}+\left\|\sigma^{1-\frac{s_{0}}{2}} \nabla^{2} v\right\|_{L_{t}^{\infty}\left(L^{2}\right)}^{2}\right) \\
& \quad \times \int_{0}^{t} \sigma^{-\left(1-\theta_{0}\right)}\left(\left\|\nabla \partial_{X}^{\ell-1} \nabla X\right\|_{L^{p}}^{2}+\left\|\partial_{X}^{\ell-1} \nabla X\right\|_{L^{\infty}}^{2}\right) \mathrm{d} t^{\prime} \\
& \quad+\langle t\rangle \sum_{l \leq \ell-2, i+j \leq \ell-1}\left(\left\|\nabla \partial_{X}^{l} \nabla X\right\|_{L_{t}^{\infty}\left(L^{p}\right)}^{2}\left\|\sigma^{1-\frac{s_{\ell-1}}{2}} \partial_{X}^{i} \nabla \mathrm{v}^{j}\right\|_{L_{t}^{\infty}\left(L^{\frac{2 p}{p-2}}\right)}^{2}\right. \\
& \left.\quad+\left\|\partial_{X}^{l} \nabla X\right\|_{L_{t}^{\infty}\left(L^{\infty}\right)}^{2}\left\|\sigma^{1-\frac{s_{\ell-1}}{2}} \nabla \partial_{X}^{i} \nabla \mathrm{v}^{j}\right\|_{L_{t}^{\infty}\left(L^{2}\right)}^{2}\right),
\end{aligned}
$$


and hence we derive from Lemmas 2.1 and 1.3, and Proposition 1.1 that

$$
\left\|\sigma^{\frac{1-s_{\ell}}{2}} \nabla \partial_{X}^{\ell} \nabla v\right\|_{L_{t}^{2}\left(L^{2}\right)}^{2} \leq \mathfrak{C}_{\ell}(X) .
$$

Similarly we derive the bound $\left\|\sigma^{\frac{1-s_{\ell}}{2}} D_{t} \partial_{X}^{\ell-1} \Delta X\right\|_{L_{t}^{2}\left(L^{2}\right)}^{2} \leq \mathfrak{C}_{\ell}(X)$ : Due to $D_{t} \mathrm{X}^{\ell-1}=\mathrm{v}^{\ell}$, we calculate the difference

$$
\begin{aligned}
D_{t} \partial_{X}^{\ell-1} \Delta X-\Delta \mathrm{v}^{\ell}= & D_{t} \sum_{i=0}^{\ell-2} \partial_{X}^{i}\left[\partial_{X} ; \Delta\right] \mathrm{X}^{\ell-2-i}+D_{t} \Delta \mathrm{X}^{\ell-1}-\Delta D_{t} \mathrm{X}^{\ell-1} \\
=- & \sum_{i=0}^{\ell-2} D_{t} \partial_{X}^{i}\left(\Delta X \cdot \nabla \mathrm{X}^{\ell-2-i}+2 \partial_{\alpha} X \cdot \nabla \partial_{\alpha} \mathrm{X}^{\ell-2-i}\right) \\
& -\left(\Delta v \cdot \nabla \mathrm{X}^{\ell-1}+2 \partial_{\alpha} v \cdot \nabla \partial_{\alpha} \mathrm{X}^{\ell-1}\right),
\end{aligned}
$$

and estimate the difference by

$$
\begin{aligned}
& \left\|\sigma^{\frac{1-s_{\ell}}{2}}\left(D_{t} \partial_{X}^{\ell-1} \Delta X-\Delta \mathrm{v}^{\ell}\right)\right\|_{L_{t}^{2}\left(L^{2}\right)}^{2} \\
& \leq C<t\rangle \sum_{m+n+\kappa+l \leq \ell-2}\left(\left\|\sigma^{1-\frac{s_{\ell-1}}{2}} D_{t} \partial_{X}^{m} \nabla^{2} X^{n}\right\|_{L_{t}^{\infty}\left(L^{2}\right)}^{2}\left\|\partial_{X}^{\kappa} \nabla X^{l}\right\|_{L_{t}^{\infty}\left(L^{\infty}\right)}^{2}\right. \\
& \left.\quad+\left\|\partial_{X}^{m} \nabla^{2} X^{n}\right\|_{L_{t}^{\infty}\left(L^{p}\right)}^{2}\left\|\sigma^{1-\frac{s_{\ell-1}}{2}} D_{t} \partial_{X}^{\kappa} \nabla X^{l}\right\|_{L_{t}^{\infty}\left(L^{\frac{2 p}{p-2}}\right)}^{2}\right)+\mathcal{C}_{0} \int_{0}^{t} \sigma^{-\left(1-\theta_{0}\right)}\left\|\nabla X^{\ell-1}\right\|_{W^{1, p}}^{2} \mathrm{~d} t^{\prime} .
\end{aligned}
$$

On the other hand, we get from the bound $A_{\ell 1}^{2} \leq \mathfrak{C}_{\ell}(X)$ and Gagliardo-Nirenberg's inequality that

$$
\left\|\sigma^{\frac{1-s_{\ell}}{p}} \nabla \mathbf{v}^{\ell}\right\|_{L_{t}^{2}\left(L^{\frac{2 p}{p-2}}\right)}^{2} \leq C\left(\left\|\nabla \mathbf{v}^{\ell}\right\|_{L_{t}^{2}\left(L^{2}\right)}^{1-\frac{2}{p}}\left\|\sigma^{\frac{1-s_{\ell}}{2}} \nabla^{2} \mathbf{v}^{\ell}\right\|_{L_{t}^{2}\left(L^{2}\right)}^{\frac{2}{p}}\right)^{2} \leq \mathfrak{C}_{\ell}(X) .
$$

Then, a similar analysis as to get (2.23) gives also $\left\|\sigma^{\frac{1-s_{\ell}}{p}} \partial_{X}^{\ell} \nabla v\right\|_{L_{t}^{2}\left(L^{\frac{2 p}{p-2}}\right)}^{2} \leq \mathfrak{C}_{\ell}(X)$. Finally the same estimate holds $\left\|\sigma^{\frac{1-s_{\ell}}{p}} D_{t} \partial_{X}^{\ell-1} \nabla X\right\|_{L_{t}^{2}\left(L^{\frac{2 p}{p-2}}\right)}^{2} \leq \mathfrak{C}_{\ell}(X)$ : since for the difference

$$
\begin{aligned}
D_{t} \partial_{X}^{\ell-1} \nabla X-\nabla \mathbf{v}^{\ell} & =D_{t} \sum_{i=0}^{\ell-2} \partial_{X}^{i}\left[\partial_{X} ; \nabla\right] \mathrm{X}^{\ell-2-i}+D_{t} \nabla \mathrm{X}^{\ell-1}-\nabla D_{t} \mathrm{X}^{\ell-1} \\
& =-\sum_{i=0}^{\ell-2} D_{t} \partial_{X}^{i}\left(\nabla X \cdot \nabla \mathrm{X}^{\ell-2-i}\right)-\nabla v \cdot \nabla \mathrm{X}^{\ell-1}
\end{aligned}
$$

we have the estimate

$$
\begin{aligned}
& \left\|\sigma^{\frac{1-s_{\ell}}{p}}\left(D_{t} \partial_{X}^{\ell-1} \nabla X-\nabla \mathbf{v}^{\ell}\right)\right\|_{L_{t}^{2}\left(L^{\frac{2 p}{p-2}}\right)}^{2} \\
\leq & \int_{0}^{t} \sigma^{-1+s_{0}-\frac{2}{p} s_{\ell}}\left\|\nabla X^{\ell-1}\right\|_{L^{\infty}}^{2} \mathrm{~d} t^{\prime}\left\|\sigma^{\frac{1}{2}+\frac{1}{p}-\frac{s_{0}}{2}} \nabla v\right\|_{L_{t}^{\infty}\left(L^{\frac{2 p}{p-2}}\right)}^{2}
\end{aligned}
$$




$$
+\langle t\rangle \sum_{m+n+\kappa+l \leq \ell-2}\left\|\sigma^{\frac{1}{2}+\frac{1}{p}-\frac{s_{\ell-1}}{2}} \partial_{X}^{m} D_{t} \nabla X^{n}\right\|_{L_{t}^{\infty}\left(L^{\frac{2 p}{p-2}}\right)}^{2}\left\|\partial_{X}^{\kappa} \nabla X^{l}\right\|_{L_{t}^{\infty}\left(L^{\infty}\right)}^{2} \leq \mathfrak{C}_{\ell}(X) .
$$

Thus, we complete the proof.

\subsection{Estimates for $\left(\mathrm{F}_{\ell}, \mathfrak{a}_{\ell}, \mathfrak{b}_{\ell}\right)$}

In this subsection we show the estimates for the data $\left(\mathrm{F}_{\ell}, \mathfrak{a}_{\ell}, \mathfrak{b}_{\ell}\right)$ in Lemmas 1.6-1.7-1.8, by use of the known estimates (1.16)-(1.38)-(1.51)-(1.52).

Proof of Lemma 1.6. Recall the definition of $\mathrm{F}_{\ell}$ in (1.32). We denote

$$
\begin{aligned}
& \widetilde{\mathrm{F}}_{\ell} \stackrel{\text { def }}{=} \mathrm{F}_{\ell}+2 \partial_{\alpha} v \cdot \nabla \partial_{\alpha} \mathrm{v}^{\ell}+\Delta v \cdot \nabla \mathrm{v}^{\ell}-\nabla v \cdot \nabla \pi^{\ell} \\
&+2 D_{t}\left(\partial_{X}^{\ell-1} \partial_{\alpha} X \cdot \nabla \partial_{\alpha} v\right)+D_{t}\left(\partial_{X}^{\ell-1} \Delta X \cdot \nabla v\right)-D_{t}\left(\partial_{X}^{\ell-1} \nabla X \cdot \nabla \pi\right),
\end{aligned}
$$

such that

$$
\begin{aligned}
\left|\widetilde{\mathrm{F}}_{\ell}\right| \leq C & \sum_{i+j \leq \ell-1, l \leq \ell-2}\left(\left|D_{t}^{2} \mathrm{v}^{i}\right|+\left|\partial_{X}^{l} D_{t} \nabla X\right|\left(\left|\partial_{X}^{i} \nabla^{2} \mathrm{v}^{j}\right|+\left|\partial_{X}^{i} \nabla \pi^{j}\right|\right)\right. \\
& \left.+\left|\partial_{X}^{l} \nabla X\right|\left(\left|\partial_{X}^{i} D_{t} \nabla^{2} \mathrm{v}^{j}\right|+\left|\partial_{X}^{i} D_{t} \nabla \pi^{j}\right|\right)+\left|\partial_{X}^{l} D_{t} \Delta X\right|\left|\partial_{X}^{i} \nabla \mathrm{v}^{j}\right|+\left|\partial_{X}^{l} \Delta X\right|\left|\partial_{X}^{i} D_{t} \nabla \mathrm{v}^{j}\right|\right) .
\end{aligned}
$$

Hence we derive from (1.38) that

$$
\begin{aligned}
\left\|\widetilde{\mathrm{F}}_{\ell}\right\|_{L^{2}}^{2} \leq \mathcal{H}_{\ell-1}^{2}(t) & \sum_{i+j \leq \ell-1}\left(\left\|\left(D_{t}^{2} \mathrm{v}^{i}, \partial_{X}^{i} D_{t} \nabla^{2} \mathrm{v}^{j}, \partial_{X}^{i} D_{t} \nabla \pi^{j}\right)\right\|_{L^{2}}^{2}+\left\|\partial_{X}^{i} D_{t} \nabla \mathrm{v}^{j}\right\|_{L^{\frac{2 p}{p-2}}}^{2}\right) \\
& +C \sum_{i+j \leq \ell-1, l \leq \ell-2}\left\|\left(\partial_{X}^{l} D_{t} \nabla X, \partial_{X}^{i} \nabla \mathrm{v}^{j}\right)\right\|_{L^{\infty}}^{2}\left\|\left(\partial_{X}^{i} \nabla^{2} \mathrm{v}^{j}, \partial_{X}^{i} \nabla \pi^{j}, \partial_{X}^{l} D_{t} \Delta X\right)\right\|_{L^{2}}^{2} \\
\leq & \mathcal{H}_{\ell-1}^{2}(t)\left(\sigma(t)^{-\left(3-s_{\ell-1}\right)} \mathfrak{B}_{\ell-1}(t)+\sigma(t)^{-\left(4-2 s_{\ell-1}\right)}\right) .
\end{aligned}
$$

As a result, we deduce from (1.38) again that

$$
\begin{aligned}
\left\|\sigma^{\frac{3-s_{\ell}}{2}} \mathrm{~F}_{\ell}\right\|_{L_{t}^{2}\left(L^{2}\right)}^{2} \leq & \mathcal{H}_{\ell}^{2}-1 \\
& +C\left\|\sigma^{\frac{3}{2}-\frac{1}{p}-\frac{s_{\ell}}{2}}\left(\nabla^{2} v, \nabla \pi\right)\right\|_{L_{t}^{\infty}\left(L^{p}\right)}^{2}\left\|\sigma^{\frac{1}{p}}\left(\nabla \mathbf{v}^{\ell}, D_{t} \partial_{X}^{\ell-1} \nabla X\right)\right\|_{L_{t}^{2}\left(L^{\frac{2 p}{p-2}}\right)}^{2}\left\|\sigma^{\frac{1-s_{\ell}}{2}}\left(\nabla^{2} \mathrm{v}^{\ell}, \nabla \pi^{\ell}, D_{t} \partial_{X}^{\ell-1} \Delta X\right)\right\|_{L_{t}^{2}\left(L^{2}\right)}^{2} \\
& +C \int_{0}^{t}\left(\left\|\partial_{X}^{\ell-1} \nabla X\right\|_{L^{\infty}}^{2}+\left\|\partial_{X}^{\ell-1} \Delta X\right\|_{L^{p}}^{2}\right) \\
& \times\left(\left\|\sigma^{\frac{3-s_{\ell}}{2}}\left(D_{t}^{2} v, D_{t} \nabla^{2} v, D_{t} \nabla \pi\right)\right\|_{L^{2}}^{2}+\left\|\sigma^{\frac{3-s_{\ell}}{2}} D_{t} \nabla v\right\|_{L^{\frac{2 p}{p-2}}}^{2}\right) \mathrm{d} t^{\prime} .
\end{aligned}
$$

By virtue of (1.38)-(1.51)-(1.52), we complete the proof of Lemma 1.6. 
Proof of Lemma 1.7. Recall the formulation of $\mathfrak{a}_{\ell}$ in Subsection 1.2.3: $\mathfrak{a}_{\ell}=\sum_{i=0}^{\ell-1} P_{X}^{i}\left(D_{t} \mathrm{v}^{\ell-1-i}\right.$. $\nabla X)+P_{X}^{\ell}(v \cdot \nabla v)$. Then we calculate

$$
\begin{aligned}
\mathfrak{a}_{\ell}= & \sum_{i=0}^{\ell-1} \sum_{i_{1}+\cdots+i_{r}=i} \partial_{X}^{i_{1}}\left(D_{t} v^{\ell-1-i} \cdot \nabla X\right) \cdot \partial_{X}^{i_{2}}(-\nabla X)^{i_{3}} \cdots \partial_{X}^{i_{r-1}}(-\nabla X)^{i_{r}} \\
& +\sum_{j_{1}+\cdots+j_{l}=\ell, j_{1} \neq \ell} \partial_{X}^{j_{1}}(v \cdot \nabla v) \cdot \partial_{X}^{j_{2}}(-\nabla X)^{j_{3}} \cdots \partial_{X}^{j_{l-1}}(-\nabla X)^{j_{l}}+\partial_{X}^{\ell}(v \cdot \nabla v),
\end{aligned}
$$

and by virtue of (1.24) such that $\operatorname{div}\left(P_{X} u\right)=\partial_{X} \operatorname{div} u$,

$$
\begin{aligned}
\operatorname{div} \mathfrak{a}_{\ell} & =\sum_{i=0}^{\ell-1} \partial_{X}^{i} \operatorname{div}\left(D_{t} \mathrm{v}^{\ell-1-i} \cdot \nabla X\right)+\partial_{X}^{\ell} \operatorname{div}(v \cdot \nabla v) \\
& =\sum_{i=0}^{\ell-1} \partial_{X}^{i}\left(\nabla D_{t} \mathrm{v}^{\ell-1-i}: \nabla X\right)+\partial_{X}^{\ell}(\nabla v: \nabla v) .
\end{aligned}
$$

Let

$$
\mathfrak{z} \ell:=\mathfrak{a}_{\ell}-\mathrm{v}^{\ell} \cdot \nabla v-v \cdot \partial_{X}^{\ell} \nabla v .
$$

Then we deduce from (1.38) and the formulation (2.27) of $\mathfrak{a}_{\ell}$ above that

$$
\begin{aligned}
\left\|\mathfrak{z}_{\ell}(t)\right\|_{L^{2}} \leq & \left(\mathcal{H}_{\ell-1}(t)+\left\|\partial_{X}^{\ell-1} \nabla X\right\|_{L^{\infty}}\right) \sigma(t)^{-\left(1-\frac{s_{\ell-1}}{2}\right)} \\
& \times \sum_{i+j \leq \ell-1}\left(\left\|\sigma^{1-\frac{s_{\ell-1}}{2}} D_{t} \mathrm{v}^{i}\right\|_{L^{2}}+\left\|\sigma^{\frac{1}{2}} \mathrm{v}^{i}\right\|_{L^{\infty}}\left\|\sigma^{\frac{1-s_{\ell-1}}{2}} \partial_{X}^{j} \nabla v\right\|_{L^{2}}\right),
\end{aligned}
$$

and hence

$$
\left\|\sigma^{\frac{1-s_{\ell}}{2}} \mathfrak{z} \ell(t)\right\|_{L^{2}}^{2} \leq \mathcal{H}_{\ell-1}^{2}(t) \sigma(t)^{-\left(1-\theta_{0}\right)}\left(1+\left\|\nabla \mathbf{X}^{\ell-1}\right\|_{W^{1, p}}^{2}\right) .
$$

To handle the estimate of $\nabla_{\mathfrak{z} \ell}$, we write

$$
\nabla \mathfrak{z}_{\ell}=\mathfrak{Z}_{\ell, 1}+\mathfrak{Z}_{\ell, 2}+\mathfrak{Z}_{\ell, 3}+\sum_{\substack{i+j=\ell \\ i \neq \ell, j \neq \ell}} \nabla\left(\mathrm{v}^{i} \cdot \partial_{X}^{j} \nabla v\right),
$$

where

$$
\begin{aligned}
& \mathfrak{Z}_{\ell, 1}:= \sum_{i=1}^{\ell-1} \sum_{i_{1}+\cdots+i_{r}=i-1}\left(\partial_{X}^{i_{1}}\left(\nabla X \cdot \nabla \partial_{X}^{i_{2}}\left(D_{t} \mathrm{v}^{\ell-1-i} \cdot \nabla X\right)\right) \cdots \partial_{X}^{i_{r-1}}(-\nabla X)^{i_{r}},\right. \\
&+\partial_{X}^{i_{1}}\left(D_{t} \mathrm{v}^{\ell-1-i} \cdot \nabla X\right) \cdots \partial_{X}^{i_{m-1}}\left(\nabla X \cdot \nabla \partial_{X}^{i_{m}}(-\nabla X)^{i_{m+1}}\right) \cdots \partial_{X}^{i_{r-1}}(-\nabla X)^{i_{r}} \\
&\left.\quad+\partial_{X}^{i_{1}}\left(D_{t} \mathrm{v}^{\ell-1-i} \cdot \nabla X\right) \cdot \partial_{X}^{i_{2}}(-\nabla X)^{i_{3}} \cdots \partial_{X}^{i_{m}} \nabla(-\nabla X) \cdots \partial_{X}^{i_{r-1}}(-\nabla X)^{i_{r}}\right), \\
& \mathfrak{Z}_{\ell, 2}:=\sum_{j_{1}+\cdots+j_{l}=\ell-1}\left(\partial_{X}^{j_{1}}\left(\nabla X \cdot \nabla \partial_{X}^{j_{2}}(v \cdot \nabla v)\right) \cdots \partial_{X}^{j_{l-1}}(-\nabla X)^{j_{l}}\right. \\
& \quad+\partial_{X}^{j_{1}}(v \cdot \nabla v) \cdots \partial_{X}^{j_{n-1}}\left(\nabla X \cdot \nabla \partial_{X}^{j_{n}}(-\nabla X)^{j_{n+1}}\right) \cdots \partial_{X}^{j_{l}-1}(-\nabla X)^{j_{l}}
\end{aligned}
$$




$$
\begin{array}{r}
\left.+\partial_{X}^{j_{1}}(v \cdot \nabla v) \cdot \partial_{X}^{j_{2}}(-\nabla X)^{j_{3}} \cdots \partial_{X}^{j_{n}} \nabla(-\nabla X) \cdots \partial_{X}^{j_{l-1}}(-\nabla X)^{j_{l}}\right), \\
\mathfrak{Z}_{\ell, 3}:=\sum_{i=0}^{\ell-1} \sum_{i_{1}+\cdots+i_{r}=i} \partial_{X}^{i_{1}} \nabla\left(D_{t} \mathrm{v}^{\ell-1-i} \cdot \nabla X\right) \cdot \partial_{X}^{i_{2}}(-\nabla X)^{i_{3}} \cdots \partial_{X}^{i_{r-1}}(-\nabla X)^{i_{r}} \\
+\sum_{j_{1}+\cdots+j_{l}=\ell, j_{1} \neq \ell} \partial_{X}^{j_{1}} \nabla(v \cdot \nabla v) \cdot \partial_{X}^{j_{2}}(-\nabla X)^{j_{3}} \cdots \partial_{X}^{j_{l-1}}(-\nabla X)^{j_{l}} .
\end{array}
$$

This gives rise to

$$
\begin{aligned}
\left\|\nabla \mathfrak{z}_{\ell}(t)\right\|_{L^{2}} \leq & \mathcal{H}_{\ell-1}(t)\left(1+\left\|\nabla X^{\ell-1}\right\|_{W^{1, p}}\right) \sum_{i+j, l \leq \ell-1}\left(\left\|\partial_{X}^{i} \nabla D_{t} \mathrm{v}^{j}\right\|_{L^{2}}+\left\|D_{t} \mathrm{v}^{i}\right\|_{L^{\frac{2 p}{p-2}}}\right. \\
& +\sigma(t)^{-\frac{3-s_{\ell-1}}{2}}\left\|\sigma^{1-\frac{s_{\ell-1}}{2}} \partial_{X}^{i} \nabla \mathbf{v}^{j}\right\|_{L^{\infty}}\left(\left\|\sigma^{\frac{1-s_{\ell-1}}{2}} \partial_{X}^{l} \nabla v\right\|_{L^{2}}+\left\|\sigma^{\frac{1}{2}} \mathbf{v}^{l}\right\|_{L^{\frac{2 p}{p-2}}}\right) \\
& \left.+\sigma(t)^{-\frac{3-s_{\ell-1}}{2}}\left\|\sigma^{\frac{1}{2}} \mathbf{v}^{l}\right\|_{L^{\infty}}\left\|\sigma^{1-\frac{s_{\ell-1}}{2}} \partial_{X}^{i} \nabla \partial_{X}^{j} \nabla v\right\|_{L^{2}}\right),
\end{aligned}
$$

from which and (1.38), we infer that

$$
\left\|\sigma^{1-\frac{s_{\ell}}{2}} \nabla_{\mathfrak{z} \ell}(t)\right\|_{L^{2}}^{2} \leq \mathcal{H}_{\ell-1}^{2}(t)\left(1+\left\|\nabla \mathbf{X}^{\ell-1}\right\|_{W^{1, p}}^{2}\right)\left(\mathfrak{B}_{\ell-1}(t)+\sigma(t)^{-\left(1-\theta_{0}\right)}\right)
$$

Taking into account of (2.28), we deduce from (2.30) and (2.32) that

$$
\begin{aligned}
&\left\|\sigma^{\frac{1-s_{\ell}}{2}} \mathfrak{a}_{\ell}\right\|_{L_{t}^{2}\left(L^{2}\right)}^{2} \leq\left\|\sigma^{\frac{1}{p}} \partial_{X}^{\ell} \nabla v\right\|_{L_{t}^{2}\left(L^{\frac{2 p}{p-2}}\right)}^{2}\left\|\sigma^{\frac{1}{2}-\frac{1}{p}-\frac{s_{0}}{2}} v\right\|_{L_{t}^{\infty}\left(L^{p}\right)}^{2}+\left\|\mathrm{v}^{\ell}\right\|_{L_{t}^{\infty}\left(L^{2}\right)}^{2}\left\|\sigma^{\frac{1-s_{\ell}}{2}} \nabla v\right\|_{L_{t}^{2}\left(L^{\infty}\right)}^{2} \\
&+\mathcal{H}_{\ell-1}^{2}(t)\left(1+\int_{0}^{t} \sigma\left(t^{\prime}\right)^{-\left(1-\theta_{0}\right)}\left\|\nabla \mathrm{X}^{\ell-1}\left(t^{\prime}\right)\right\|_{W^{1, p}}^{2} \mathrm{~d} t^{\prime}\right) \\
&\left\|\sigma^{1-\frac{s_{\ell}}{2}} \nabla \mathfrak{a}_{\ell}\right\|_{L_{t}^{2}\left(L^{2}\right)}^{2} \leq\left\|\sigma^{\frac{1-s_{\ell}}{p}}\left(\nabla \mathrm{v}^{\ell}, \partial_{X}^{\ell} \nabla v\right)\right\|_{L_{t}^{2}\left(L^{\frac{2 p}{p-2}}\right)}^{2}\left\|\sigma^{1-\frac{1}{p}-\frac{s_{0}}{2}} \nabla v\right\|_{L_{t}^{\infty}\left(L^{p}\right)}^{2} \\
&+\left\|\mathrm{v}^{\ell}\right\|_{L_{t}^{4}\left(L^{4}\right)}^{2}\left\|\sigma^{1-\frac{s_{\ell}}{2}} \nabla^{2} v\right\|_{L_{t}^{4}\left(L^{4}\right)}^{2}+\left\|\sigma^{\frac{1}{2}} v\right\|_{L_{t}^{\infty}\left(L^{\infty}\right)}^{2}\left\|\sigma^{\frac{1-s_{\ell}}{2}} \nabla \partial_{X}^{\ell} \nabla v\right\|_{L_{t}^{2}\left(L^{2}\right)}^{2} \\
&+\mathcal{H}_{\ell-1}^{2}(t)\left(1+\int_{0}^{t}\left(\mathfrak{B}_{\ell-1}\left(t^{\prime}\right)+\sigma\left(t^{\prime}\right)^{-\left(1-\theta_{0}\right)}\right)\left\|\nabla X^{\ell-1}\left(t^{\prime}\right)\right\|_{W^{1, p}}^{2} \mathrm{~d} t^{\prime}\right) .
\end{aligned}
$$

Together with (1.16)-(1.51)-(1.52), we conclude

$$
\begin{aligned}
& \left\|\sigma^{\frac{1-s_{\ell}}{2}} \mathfrak{a}_{\ell}\right\|_{L_{t}^{2}\left(L^{2}\right)}^{2}+\left\|\sigma^{1-\frac{s_{\ell}}{2}} \nabla \mathfrak{a}_{\ell}\right\|_{L_{t}^{2}\left(L^{2}\right)}^{2} \\
\leq & \mathcal{H}_{\ell-1}^{2}(t)\left(\mathcal{C}_{\ell}+\int_{0}^{t}\left(\mathfrak{B}_{\ell-1}\left(t^{\prime}\right)+\sigma\left(t^{\prime}\right)^{-\left(1-\frac{\theta_{0}}{2}\right)}\right)\left\|\nabla X^{\ell-1}\left(t^{\prime}\right)\right\|_{W^{1, p}}^{2} \mathrm{~d} t^{\prime}\right) .
\end{aligned}
$$


Finally let us turn to the estimate of $\nabla \operatorname{div} \mathfrak{a}_{\ell}$ :

$$
\begin{aligned}
& \left\|\sigma^{\frac{3-s_{\ell}}{2}} \nabla \operatorname{div} \mathfrak{a}_{\ell}\right\|_{L_{t}^{2}\left(L^{2}\right)} \\
& \leq\left(\int_{0}^{t}\left(\left\|\sigma^{\frac{3-s_{\ell}}{2}} \nabla^{2} D_{t} v\right\|_{L^{2}}^{2}+\left\|\sigma^{\frac{3-s_{\ell}}{2}} \nabla D_{t} v\right\|_{L^{\frac{2 p}{p-2}}}^{2}\right)\left\|\partial_{X}^{\ell-1} \nabla X\right\|_{W^{1, p}}^{2} d t^{\prime}\right)^{\frac{1}{2}} \\
& \quad+C R_{\ell-1}(t) \sum_{i+j \leq \ell-1}\left(\left\|\sigma^{\frac{3-s_{\ell}}{2}} \nabla \partial_{X}^{i} \nabla D_{t} \mathrm{v}^{j}\right\|_{L_{t}^{2}\left(L^{2}\right)}+\left\|\sigma^{\frac{3-s_{\ell}}{2}} \partial_{X}^{i} \nabla D_{t} \mathrm{v}^{j}\right\|_{L_{t}^{2}\left(L^{\left.\frac{2 p}{p-2}\right)}\right.}\right) \\
& \quad+\left\|\sigma^{\frac{3}{2}-\frac{1}{p}-\frac{s_{0}}{2}} \nabla^{2} v\right\|_{L_{t}^{\infty}\left(L^{p}\right)}\left\|\sigma^{\frac{1}{p}} \partial_{X}^{\ell} \nabla v\right\|_{L_{t}^{2}\left(L^{\frac{2 p}{p-2}}\right)}+\|\sigma \nabla v\|_{L_{t}^{\infty}\left(L^{\infty}\right)}\left\|\sigma^{\frac{1-s_{\ell}}{2}} \nabla \partial_{X}^{\ell} \nabla v\right\|_{L_{t}^{2}\left(L^{2}\right)} \\
& \quad+\sum_{i, j \leq \ell-1}\left\|\sigma^{\frac{3}{2}-\frac{1}{p}-\frac{s_{\ell-1}}{2}} \nabla \partial_{X}^{i} \nabla v\right\|_{L_{t}^{\infty}\left(L^{p}\right)}\left\|\sigma^{\frac{1}{2}+\frac{1}{p}} \partial_{X}^{j} \nabla v\right\|_{L_{t}^{\infty}\left(L^{\left.\frac{2 p}{p-2}\right)}\right.}\left\|\sigma^{-\frac{1-\theta_{0}}{2}}\right\|_{L^{2}([0, t])} .
\end{aligned}
$$

Then, by virtue of Eqs. (1.16), (1.38), (1.51), (1.52) and (2.33), we complete the proof of (1.53).

Proof of Lemma 1.8. Recall the formulation $\mathfrak{b}_{\ell}$ given in Subsection 1.2.3:

$$
\begin{aligned}
\mathfrak{b}_{\ell} & =\sum_{i=0}^{\ell-1} P_{X}^{i}\left(D_{t}^{2} \mathbf{v}^{\ell-1-i} \cdot \nabla X\right)+P_{X}^{\ell} \mathfrak{b}_{0} \\
& =\sum_{i=0}^{\ell-1} \sum_{i_{1}+\cdots+i_{r}=i} \partial_{X}^{i_{1}}\left(D_{t}^{2} \mathbf{v}^{\ell-1-i} \cdot \nabla X\right) \cdots \partial_{X}^{i_{r-1}}(-\nabla X)^{i_{r}}+\sum_{j_{1}+\cdots+j_{l}=\ell} \partial_{X}^{j_{1}} \mathfrak{k}_{0} \cdots \partial_{X}^{j_{l}-1}(-\nabla X)^{j_{l}},
\end{aligned}
$$

where

$$
\mathfrak{b}_{0}=v \cdot\left(\nabla v_{t}+D_{t} \nabla v\right)+D_{t} v \cdot \nabla v=2 v \cdot D_{t} \nabla v-v \cdot \nabla(v \cdot \nabla v)+D_{t} v \cdot \nabla v .
$$

We calculate directly

$$
\begin{aligned}
& \quad\left\|\sigma^{\frac{3-s_{\ell}}{2}} \mathfrak{b}_{\ell}\right\|_{L_{t}^{2}\left(L^{2}\right)}^{2} \\
& \leq \int_{0}^{t}\left\|\sigma^{\frac{3-s_{\ell}}{2}}\left(D_{t}^{2} v, \mathfrak{b}_{0}\right)\right\|_{L^{2}}^{2}\left\|\nabla \mathbf{X}^{\ell-1}\right\|_{L^{\infty}}^{2} \mathrm{~d} t^{\prime} \\
& \quad+2\left\|\sigma^{\frac{1}{2}} v\right\|_{L_{t}^{\infty}\left(L^{\infty}\right)}^{2}\left\|\sigma^{1-\frac{s_{\ell}}{2}} \partial_{X}^{\ell} D_{t} \nabla v\right\|_{L_{t}^{2}\left(L^{2}\right)}^{2}+\|\sigma \nabla v\|_{L_{t}^{\infty}\left(L^{\infty}\right)}^{2}\left\|\sigma^{\frac{1-s_{\ell}}{2}} D_{t} \mathrm{v}^{\ell}\right\|_{L_{t}^{2}\left(L^{2}\right)}^{2} \\
& \quad+C\left\|\sigma^{\frac{1}{p}} \mathbf{v}^{\ell}\right\|_{L_{t}^{\infty}\left(L^{\frac{2 p}{p-2}}\right)}^{2}\left\|\sigma^{\frac{3}{2}-\frac{1}{p}-\frac{s_{0}}{2}}\left(D_{t} \nabla v, \nabla(v \otimes \nabla v)\right)\right\|_{L_{t}^{2}\left(L^{p}\right)}^{2} \\
& \quad+\left\|\sigma^{\frac{1}{p}} \partial_{X}^{\ell} \nabla v\right\|_{L_{t}^{2}\left(L^{\frac{2 p}{p-2}}\right)}^{2}\left\|\sigma^{\frac{3}{2}-\frac{1}{p}-\frac{s_{0}}{2}}\left(v \otimes \nabla v, D_{t} v\right)\right\|_{L_{t}^{\infty}\left(L^{p}\right)}^{2} \\
& \quad+C\left(1+R_{\ell-1}(t)\right)^{4} \sum_{i, j \leq \ell-1}\left(\langle t\rangle\left\|\sigma^{1-\frac{s_{\ell-1}}{2}} D_{t} \mathrm{v}^{i}\right\|_{L_{t}^{\infty}\left(L^{2}\right)}^{2}\left\|\sigma \partial_{X}^{j} \nabla v\right\|_{L_{t}^{\infty}\left(L^{\infty}\right)}^{2}\right. \\
& \left.\quad+\left\|\sigma^{\frac{3-s_{\ell}}{2}} D_{t}^{2} \mathrm{v}^{i}\right\|_{L_{t}^{2}\left(L^{2}\right)}^{2}+\left\|\sigma \mathbf{v}^{i}\right\|_{L_{t}^{\infty}\left(L^{\infty}\right)}^{2}\left\|\sigma^{1-\frac{s_{\ell}}{2}}\left(\partial_{X}^{j} \nabla(v \cdot \nabla v), D_{t} \partial_{X}^{j} \nabla v\right)\right\|_{L_{t}^{2}\left(L^{2}\right)}^{2}\right) .
\end{aligned}
$$


Noticing the commutator

$$
\partial_{X}^{\ell} D_{t} \nabla v-\nabla D_{t} \mathrm{v}^{\ell}=\partial_{X}^{\ell}\left[D_{t} ; \nabla\right] v+\sum_{i=0}^{\ell-1} \partial_{X}^{i}\left[\partial_{X} ; \nabla\right] D_{t} \mathrm{v}^{\ell-1-i},
$$

we obtain

$$
\begin{aligned}
& \left\|\sigma^{1-\frac{s_{\ell}}{2}} \partial_{X}^{\ell} D_{t} \nabla v\right\|_{L_{t}^{2}\left(L^{2}\right)} \\
\leq & \left\|\sigma^{1-\frac{s_{\ell}}{2}} \nabla D_{t} \mathrm{v}^{\ell}\right\|_{L_{t}^{2}\left(L^{2}\right)}+2\left\|\sigma^{\frac{1}{p}} \partial_{X}^{\ell} \nabla v\right\|_{L_{t}^{2}\left(L^{\frac{2 p}{p-2}}\right)}\left\|\sigma^{1-\frac{1}{p}-\frac{s_{0}}{2}} \nabla v\right\|_{L_{t}^{\infty}\left(L^{p}\right)} \\
& +\left(\int_{0}^{t}\left\|\sigma^{1-\frac{s_{\ell}}{2}} \nabla D_{t} v\right\|_{L^{2}}^{2}\left\|\nabla X^{\ell-1}\right\|_{L^{\infty}}^{2} d t^{\prime}\right)^{\frac{1}{2}} \\
& +\sum_{i, j \leq \ell-1}\left\|\sigma \partial_{X}^{i} \nabla v\right\|_{L_{t}^{\infty}\left(L^{\infty}\right)}\left\|\sigma^{\frac{1-s_{\ell-1}}{2}} \partial_{X}^{j} \nabla v\right\|_{L_{t}^{\infty}\left(L^{2}\right)}\left\|\sigma^{-\frac{1-\theta_{0}}{2}}\right\|_{L^{2}([0, t])} \\
& +C\left(R_{\ell-1}(t)+R_{\ell-1}^{2}(t)\right) \sum_{i+j \leq \ell-1}\left\|\sigma^{1-\frac{s_{\ell}}{2}} \partial_{X}^{i} \nabla D_{t} \mathrm{v}^{j}\right\|_{L_{t}^{2}\left(L^{2}\right)^{\prime}}
\end{aligned}
$$

from which, (1.16), (1.38) and (1.52), we infer

$$
\begin{aligned}
& \left\|\sigma^{1-\frac{s_{\ell}}{2}} \partial_{X}^{\ell} D_{t} \nabla v\right\|_{L_{t}^{2}\left(L^{2}\right)}^{2} \\
\leq & \left\|\sigma^{1-\frac{s_{\ell}}{2}} \nabla D_{t} \mathrm{v}^{\ell}\right\|_{L_{t}^{2}\left(L^{2}\right)}^{2}+\mathfrak{C}_{\ell}(X)+\int_{0}^{t}\left\|\sigma^{1-\frac{s_{\ell}}{2}} \nabla D_{t} v\right\|_{L^{2}}^{2}\left\|\nabla X^{\ell-1}\right\|_{L^{\infty}}^{2} \mathrm{~d} t^{\prime} .
\end{aligned}
$$

Recall the estimate (1.36) in Lemma 1.2, we know from the $D_{t} \mathrm{v}^{\ell}$-equation (1.32) that

$$
\begin{aligned}
& \left\|\sigma^{1-\frac{s_{\ell}}{2}} \nabla D_{t} \mathrm{v}^{\ell}\right\|_{L_{t}^{2}\left(L^{2}\right)}^{2} \\
\leq & \varepsilon\left\|\sigma^{\frac{3-s_{\ell}}{2}} D_{t}^{2} \mathbf{v}^{\ell}\right\|_{L_{t}^{2}\left(L^{2}\right)}^{2}+C_{\varepsilon}\left(\left\|\sigma^{\frac{1-s_{\ell}}{2}}\left(D_{t} \mathrm{v}^{\ell}, \mathfrak{a}_{\ell}\right)\right\|_{L_{t}^{2}\left(L^{2}\right)}^{2}+\left\|\sigma^{1-\frac{s_{\ell}}{2}} \nabla \mathfrak{a}_{\ell}\right\|_{L_{t}^{2}\left(L^{2}\right)}^{2}\right. \\
& \left.+\left\|\sigma^{\frac{3-s_{\ell}}{2}}\left(\mathrm{~F}_{\ell}, \nabla \operatorname{div} \mathfrak{a}_{\ell}\right)\right\|_{L_{t}^{2}\left(L^{2}\right)}^{2}\right) .
\end{aligned}
$$

Inserting the above estimate into (2.35) and using Lemmas 1.6 and 1.7, we achieve

$$
\left\|\sigma^{1-\frac{s_{\ell}}{2}} \partial_{X}^{\ell} D_{t} \nabla v\right\|_{L_{t}^{2}\left(L^{2}\right)}^{2} \leq \varepsilon\left\|\sigma^{\frac{3-s_{\ell}}{2}} D_{t}^{2} \mathbf{v}^{\ell}\right\|_{L_{t}^{2}\left(L^{2}\right)}^{2}+C_{\varepsilon} \widetilde{\mathfrak{C}}_{\ell}(X)
$$

with $\widetilde{\mathfrak{C}}_{\ell}(X)$ defined in (1.54).

Finally, we notice that

$$
\begin{aligned}
& \sum_{j \leq \ell-1}\left\|\sigma^{1-\frac{s_{\ell}}{2}} \partial_{X}^{j} \nabla(v \cdot \nabla v)\right\|_{L_{t}^{2}\left(L^{2}\right)} \\
& \leq C\langle t\rangle^{\frac{1}{2}} \sum_{i+l \leq \ell-1}\left(\left\|\sigma^{\frac{1}{2}} \mathrm{v}^{i}\right\|_{L_{t}^{\infty}\left(L^{\infty}\right)}\left\|\sigma^{1-\frac{s_{\ell-1}}{2}} \partial_{X}^{l} \nabla^{2} v\right\|_{L_{t}^{\infty}\left(L^{2}\right)}\right. \\
&\left.+\left\|\sigma^{1-\frac{s_{\ell-1}}{2}} \partial_{X}^{i} \nabla v\right\|_{L_{t}^{\infty}\left(L^{\infty}\right)}\left\|\sigma^{\frac{1}{2}} \partial_{X}^{l} \nabla v\right\|_{L_{t}^{\infty}\left(L^{2}\right)}\right) .
\end{aligned}
$$

Substituting the above inequality and (2.37) into (2.34) and using (1.16), (1.38) and (1.52), we conclude the proof of (1.55). 


\subsection{Proof of Proposition 1.2}

We prove in this subsection Proposition 1.2.

Proof of Proposition 1.2. Recall the definition of $A_{\ell 2}$ in (1.14a). We first get, by applying Lemma 1.2 to the linear system (1.32), that

$$
A_{\ell 2}^{2}(t) \leq \mathcal{C}_{0}\left(\left\|\sigma^{\frac{1-s_{\ell}}{2}}\left(D_{t} \mathrm{v}^{\ell}, \mathfrak{a}_{\ell}\right)\right\|_{L_{t}^{2}\left(L^{2}\right)}^{2}+\left\|\sigma^{1-\frac{s_{\ell}}{2}} \nabla \mathfrak{a}_{\ell}\right\|_{L_{t}^{2}\left(L^{2}\right)}^{2}+\left\|\sigma^{\frac{3-s_{\ell}}{2}}\left(\nabla \operatorname{div} \mathfrak{a}_{\ell}, \mathfrak{b}_{\ell}, \mathrm{F}_{\ell}\right)\right\|_{L_{t}^{2}\left(L^{2}\right)}^{2}\right),
$$

from which, Proposition 1.1, Corollary 1.1 and Lemmas 1.6, 1.7 and 1.8, we infer

$$
A_{\ell 1}^{2}(t)+A_{\ell 2}^{2}(t) \leq C \varepsilon\left\|\sigma^{\frac{3-s_{\ell}}{2}} D_{t}^{2} \mathbf{v}^{\ell}\right\|_{L_{t}^{2}\left(L^{2}\right)}^{2}+C_{\varepsilon} \widetilde{\mathfrak{C}}_{\ell}(X),
$$

for $\widetilde{\mathfrak{C}}_{\ell}(X)$ given by (1.54). Taking $\varepsilon$ so small that $C \varepsilon \leq \frac{1}{2}$ ensures $A_{\ell 1}^{2}(t)+A_{\ell 2}^{2}(t) \leq \widetilde{\mathfrak{C}}_{\ell}(X)$, and hence by Gagliardo-Nirenberg's inequality

$$
\left\|\sigma^{1-\frac{s_{\ell}}{2}} D_{t} \mathrm{v}^{\ell}\right\|_{L_{t}^{\frac{2 r}{r-2}\left(L^{r}\right)}} \leq \widetilde{\mathfrak{C}}_{\ell}^{\frac{1}{2}}(X),
$$

which together with the fact: $p<2 /\left(1-s_{\ell}\right)$, ensures that

$$
\left\|D_{t} \mathbf{v}^{\ell}\right\|_{L_{t}^{1}\left(L^{p}\right)}=\left\|\sigma^{1-\frac{s_{\ell}}{2}} D_{t} \mathbf{v}^{\ell}\right\|_{L_{t}^{\frac{2 p}{p-2}}\left(L^{p}\right)}\left\|\sigma^{-1+\frac{s_{\ell}}{2}}\right\|_{L^{\frac{2 p}{p+2}}([0, t])} \leq \widetilde{\mathfrak{C}}_{\ell}^{\frac{1}{2}}(X) .
$$

Recall the Eq. (1.12): $D_{t} \mathrm{X}^{\ell-1}=\mathrm{v}^{\ell}=X \cdot \nabla \mathrm{v}^{\ell-1}$. We first get the $L^{p}$ type energy estimate for $\mathbf{X}^{\ell-1}$

$$
\left\|\mathrm{X}^{\ell-1}(t)\right\|_{L^{p}} \leq\left\|\partial_{X_{0}}^{\ell-1} X_{0}\right\|_{L^{p}}+\langle t\rangle\|X\|_{L_{t}^{\infty}\left(L^{\infty}\right)}\left\|\sigma^{1-\frac{s_{\ell-1}}{2}} \nabla \mathrm{v}^{\ell-1}\right\|_{L_{t}^{\infty}\left(L^{p}\right)} \leq \mathcal{C}_{\ell}+\mathcal{H}_{\ell-1}(t) .
$$

We then apply $\Delta$ to (1.12) to obtain the $L^{p}$ type estimate for $\Delta \mathrm{X}^{\ell-1}$

$$
\left\|\Delta \mathrm{X}^{\ell-1}(t)\right\|_{L^{p}} \leq\left\|\Delta \partial_{X_{0}}^{\ell-1} X_{0}\right\|_{L^{p}}+\int_{0}^{t}\left(\|\Delta v\|_{L^{p}}+\|\nabla v\|_{L^{\infty}}\right)\left\|\nabla X^{\ell-1}\right\|_{W^{1, p}} \mathrm{~d} t^{\prime}+\left\|\Delta \mathrm{v}^{\ell}\right\|_{L_{t}^{1}\left(L^{p}\right)} .
$$

We sum up the above two inequalities and then apply Gronwall's inequality to obtain

$$
\left\|\mathrm{X}^{\ell-1}\right\|_{L_{t}^{\infty}\left(W^{2, p}\right)} \leq \exp \left(\mathcal{C}_{0} t\right)\left(\mathcal{C}_{\ell}+\mathcal{H}_{\ell-1}(t)+\left\|\Delta \mathrm{v}^{\ell}\right\|_{L_{t}^{1}\left(L^{p}\right)}\right),
$$

which together with the second inequality of (1.44) ensures that

$$
\left\|\mathrm{X}^{\ell-1}\right\|_{L_{t}^{\infty}\left(W^{2, p}\right)} \leq \mathcal{H}_{\ell-1}(t)\left(\mathcal{C}_{\ell}+\left\|D_{t} \mathrm{v}^{\ell}\right\|_{L_{t}^{1}\left(L^{p}\right)}+\int_{0}^{t} \sigma^{-\left(\frac{3}{2}-\frac{1}{p}-\frac{s_{\ell}}{2}\right)}\left\|\nabla \mathrm{X}^{\ell-1}\right\|_{W^{1, p}} \mathrm{~d} t^{\prime}\right) .
$$

Inserting (2.39) into the above inequality and using the fact that (noticing also $\frac{1}{p}>\frac{1-s_{\ell}}{2}$ )

$$
\int_{0}^{t} \sigma^{-\left(\frac{3}{2}-\frac{1}{p}-\frac{s_{\ell-1}}{2}\right)}\left\|\nabla X^{\ell-1}\right\|_{W^{1, p}} \mathrm{~d} t^{\prime} \leq\langle t\rangle^{\frac{1}{2}}\left(\int_{0}^{t} \sigma^{-\left(1-\frac{\theta_{0}}{2}\right)}\left\|\nabla X^{\ell-1}\right\|_{W^{1, p}}^{2} \mathrm{~d} t^{\prime}\right)^{\frac{1}{2}},
$$


we infer $\left\|X^{\ell-1}\right\|_{L_{t}^{\infty}\left(W^{2, p}\right)}^{2} \leq \widetilde{\mathfrak{C}}_{\ell}(X)$. Taking into account of the definition of $\widetilde{\mathfrak{C}}_{\ell}(X)$ given by (1.54), we get, by applying Gronwall's inequality and (1.38), that

$$
\left\|X^{\ell-1}\right\|_{L_{t}^{\infty}\left(W^{2, p}\right)} \leq \mathcal{H}_{\ell}(t) .
$$

Hence $\widetilde{\mathfrak{C}}_{\ell}(X) \leq \mathcal{H}_{\ell}(t)$ and (1.56) follows.

\section{Acknowledgements}

We would like to thank Professor Jean-Yves Chemin and Professor Raphaël Danchin for inspiring discussions of this topic. Part of this work was done when we were visiting Morningside Center of Mathematics (MCM), Chinese Academy of Sciences. We thank MCM for the hospitality and the financial support. X. Liao is supported by SFB 1060, Universität Bonn during the last part of the work. P. Zhang is partially supported by NSF of China under Grants Nos. 11371347 and 11688101, and innovation grant from National Center for Mathematics and Interdisciplinary Sciences.

\section{References}

[1] H. Abidi, Équations de Navier-Stokes avec densité et viscosité variables dans l'espace critique, Rev. Mat. Iberoam., 23 (2007), 537-586.

[2] H. Abidi and M. Paicu, Existence globale pour un fluide inhomogène, Ann. Inst. Fourier, 57 (2007), 883-917.

[3] H. Abidi, G. Gui and P. Zhang, On the wellposedness of 3-D inhomogeneous Navier-Stokes equations in the critical spaces, Arch. Ration. Mech. Anal., 204 (2012), 189-230.

[4] H. Abidi, G. Gui and P. Zhang, Wellposedness of 3-D inhomogeneous Navier-Stokes equations with highly oscillating initial velocity field, J. Math. Pures Appl., 100 (2013), 166-203.

[5] H. Bahouri, J.-Y. Chemin and R. Danchin, Fourier Analysis and Nonlinear Partial Differential Equations, Grundlehren der mathematischen Wissenschaften, Springer, 2010.

[6] A.-L. Bertozzi and P. Constantin, Global regularity for vortex patches, Commun. Math. Phys., 152 (1993), 19-28.

[7] J.-Y. Chemin, Calcul paradifférentiel précisé et applications à des équations aux dérivées partielles non semilinéaires, Duke Math. J., 56 (1988), 431-469.

[8] J.-Y. Chemin, Sur le mouvement des particules d'un fluide parfait incompressible bidimensionnel, Invent. Math., 103 (1991), 599-629.

[9] J.-Y. Chemin, Persistance de structures géométriques dans les fluides incompressibles bidimensionnels, Ann. Sci. École Norm. Sup., 26 (1993), 517-542.

[10] J.-Y. Chemin, Perfect incompressible fluids, Oxford Lecture Series in Mathematics and its Applications, 14. The Clarendon Press, Oxford University Press, New York, 1998.

[11] R. Danchin, Poches de tourbillon visqueuses, J. Math. Pures Appl., 76 (1997), 609-647.

[12] R. Danchin, Density-dependent incompressible viscous fluids in critical spaces, Proc. Roy. Soc. Edinburgh Sect. A, 133 (2003), 1311-1334.

[13] R. Danchin and P.-B. Mucha, A Lagrangian approach for the incompressible Navier-Stokes equations with variable density, Commun. Pure Appl. Math., 65 (2012), 1458-1480. 
[14] R. Danchin and P.-B. Mucha, Incompressible flows with piecewise constant density, Arch. Ration. Mech. Anal., 207 (2013), 991-1023.

[15] R. Danchin and P.-B. Mucha, The incompressible Navier-Stokes equations in vacuum, arXiv:1705.06061.

[16] R. Danchin and P. Zhang, Inhomogeneous Navier-Stokes equations in the half-space, with only bounded density, J. Funct. Anal., 267 (2014), 2371-2436.

[17] R. Danchin and X. Zhang, On the persistence of Hölder regular patches of density for the inhomogeneous Navier-Stokes equations, J. Éc. Polytech. Math., 4 (2017), 781-811.

[18] P. Gamblin and X. Saint-Raymond, On three-dimensional vortex patches, Bull. Soc. Math. France, 123 (1995), 375-424.

[19] F. Gancedo and E. Garcia-Juarez, Global regularity of 2D density patches for inhomogeneous Navier-Stokes, Arch. Ration. Mech. Anal., 229 (2018), 339-360.

[20] T. Hmidi, Régularité Höldérienne des poches de tourbillon visqueuses, J. Math. Pures Appl., 84 (2005), 1455-1495.

[21] T. Hmidi, Poches de tourbillon singulières dans un fluide faiblement visqueux, Rev. Mat. Iberoam., 22 (2006), 489-543.

[22] J. Huang, M. Paicu and P. Zhang, Global well-posedness of incompressible inhomogeneous fluid systems with bounded density or non-Lipschitz velocity, Arch. Ration. Mech. Anal., 209 (2013), 631-682.

[23] D. Hoff, Global solutions of the Navier-Stokes equations for multidimensional compressible flow with discontinuous initial data, J. Differential Equations, 120 (1995), 215-254.

[24] O.-A. Ladyzhenskaja and V.-A. Solonnikov, The unique solvability of an initial-boundary value problem for viscous incompressible inhomogeneous fluids. (Russian) Boundary value problems of mathematical physics, and related questions of the theory of functions, 8, Zap. Naucn. Sem. Leningrad. Otdel. Mat. Inst. Steklov. (LOMI), 52 (1975), 218-219.

[25] X. Liao and P. Zhang, On the global regularity of two-dimensional density patch for inhomogeneous incompressible viscous flow, Arch. Ration. Mech. Anal., 220 (2016), 937-981.

[26] X. Liao and P. Zhang, Global regularity of 2-D density patches for viscous inhomogeneous incompressible flow with general density: low regularity case, Accepted by Commun. Pure Appl. Math., 72 (2019), 835-884. https://onlinelibrary.wiley.com/toc/10970312/2019/72/4.

[27] X. Liao and P. Zhang, Global regularities of two-dimensional density patch for inhomogeneous incompressible viscous flow with general density, arXiv:1604.07922.

[28] P.-L. Lions, Mathematical topics in fluid mechanics. Vol. 1. Incompressible models, Oxford Lecture Series in Mathematics and Its Applications, 3. Oxford Science Publications, The Clarendon Press, Oxford University Press, New York, 1996.

[29] M. Paicu and P. Zhang, Global solutions to the 3-D incompressible inhomogeneous NavierStokes system, J. Funct. Anal., 262 (2012), 3556-3584.

[30] M. Paicu, P. Zhang and Z. Zhang, Global unique solvability of inhomogeneous NavierStokes equations with bounded density, Commun. Partial Differential Equations, 38 (2013), 1208-1234.

[31] P. Zhang and Q. Qiu, Propagation of higher-order regularities of the boundaries of 3-D vortex patches, Chinese Ann. Math. Ser. A, 18 (1997), 381-390.

[32] J. Simon, Nonhomogeneous viscous incompressible fluids: existence of velocity, density, and pressure, SIAM J. Math. Anal., 21 (1990), 1093-1117. 